\title{
Traffic grooming in bidirectional WDM ring networks*
}

\author{
Jean-Claude Bermond ${ }^{1}$ \\ Xavier Muñoz ${ }^{2}$ \\ Jean-Claude.Bermond@sophia.inria.fr xml@ma4.upc.edu \\ ignasi.sau@gmail.com
}

Version of June 3, 2010

\begin{abstract}
We study the minimization of ADMs (Add-Drop Multiplexers) in optical WDM bidirectional rings considering symmetric shortest path routing and all-to-all unitary requests. We precisely formulate the problem in terms of graph decompositions, and state a general lower bound for all the values of the grooming factor $C$ and $N$, the size of the ring. We first study exhaustively the cases $C=1, C=2$, and $C=3$, providing improved lower bounds, optimal constructions for several infinite families, as well as asymptotically optimal constructions and approximations. We then study the case $C>3$, focusing specifically on the case $C=k(k+1) / 2$ for some $k \geq 1$. We give optimal decompositions for several congruence classes of $N$ using the existence of some combinatorial designs. We conclude with a comparison of the cost functions in unidirectional and bidirectional WDM rings.
\end{abstract}

Keywords: Traffic grooming, SONET ADM, optical WDM network, graph decomposition, combinatorial designs.

\section{Introduction}

\subsection{Background and motivation}

Optical wavelength division multiplexing (WDM) is today the most promising technology to accommodate the explosive growth of Internet and telecommunication traffic in wide-area, metro-area, and backbone networks. Using WDM, the potential bandwidth of approximately $50 \mathrm{THz}$ of a fiber can be divided into multiple non-overlapping wavelength or frequency channels. Since currently the commercially available optical fibers can support over a hundred frequency channels, such a channel has over one gigabit-per-second transmission speed. However, the network is usually required to support traffic

\footnotetext{
${ }^{1}$ Mascotte joint Project I3S (CNRS/UNS) and INRIA - 2004, route des Lucioles - Sophia Antipolis, France.

${ }^{2}$ Graph Theory and Combinatorics group at Applied Mathematics IV Department of UPC - Barcelona, Spain.

*An extended abstract of this article was presented at ICTON'06 [9]. This work was partially supported by European project IST FET AEOLUS, by the Ministerio de Educación y Ciencia, Spain, and the European Regional Development Fund under project TEC2005-03575, by the Catalan Research Council under project 2005 SGR00256 and by COST action 293.
} 
connections at rates that are lower than the full wavelength capacity. In order to save equipment cost and improve network performance, it turns out to be very important to aggregate the multiple low-speed traffic connections, namely requests, into higher speed streams. Traffic grooming is the term used to carry out this aggregation, while optimizing the equipment cost.

Among possible criteria to minimize the equipment cost, one is to minimize the number of wavelengths used to route all the requests $[2,20]$. A better approximation of the true equipment cost is to minimize the number of add/drop locations, namely ADMs using SONET terminology, instead of the number of wavelengths. This leads to the grooming problem, that we state formally later in Section 2. These two problems are proved to be different. Indeed, it is known that even for a simple network like the unidirectional ring, the number of wavelengths and the number of ADMs cannot be simultaneously minimized [12,23].

The SONET ring is the most widely used optical network infrastructure today. In these networks, a communication between a pair of nodes is done via a lightpath, and each lightpath uses an AddDrop Multiplexer $(A D M)$, i.e., an electronic termination, at each of its two endpoints (but none in the intermediate nodes). If each request uses $\frac{1}{C}$ of the capacity of a wavelength, then $C$ is said to be the grooming factor, i.e., $C$ requests can be aggregated in the same wavelength through the same link. If two or more lightpaths using the same wavelength share a common endpoint, then the same ADM might be used for all lightpaths and therefore the number of ADMs needed could be reduced. Due to this fact, it makes sense to try to minimize the total number of ADMs required.

\subsection{Previous work and our contribution}

The notion of traffic grooming was introduced in [25] for the ring topology. Since then, traffic grooming has been widely studied in the literature (cf. [22,29,35] for some surveys). The problem has been proved to be NP-complete for ring networks and general $C$ [12]. Hardness results for rings and paths have been proved in [1]. Many heuristics have been proposed, but exact solutions have been found only for certain values of $C$ and for the uniform all-to-all traffic case in unidirectional ring and path topologies [8].

Many versions of the problem can be considered, according for example to the routing, the physical graph, and the request graph, among others. For example, in $[3,6]$ the Path Traffic Grooming problem is studied. If the network topology is a ring (which is the case of SONET rings), we mainly distinguish two cases depending on the routing. The Unidirectional Ring Traffic Grooming problem has been studied extensively in the literature. In an unidirectional ring, requests are routed following only one direction in the cycle. To date, the all-to-all case has been completely solved for values of the grooming factor up to $8[4,5,8,16,17]$. Also, recently the unidirectional ring with bounded degree request graph has been studied $[28,30]$.

In the Bidirectional Ring Traffic Grooming problem, the scenario is quite different. In a bidirectional ring, requests are routed either clockwise or counterclockwise. This case has been much less studied than the unidirectional one, due to its higher complexity. There is important work providing 
heuristics for the ring traffic grooming [11, 12, 20,21,23,24,27,31], but there is still an important lack of theoretical analysis of the problem. Nevertheless, its study has attracted the interest of numerous researchers. For instance, in [26] a MILP formulation of the problem can be found. In [33] two lower bounds are provided for the number of ADMs in a bidirectional ring with traffic grooming, and in [14] another lower bound is proved, regardless of the routing. In [18, 19,32,33] tools from design theory are applied to the bidirectional ring. Their method is based in the idea of primitive rings, which consists roughly in appropriately generating subgraphs of the request graph inducing unitary load each, and then packing them into sets of at most $C$ subgraphs. Namely, in [33] several heuristics are proposed, the cases $C=2$ and $C=4$ are studied in [32] (as well as other solutions that do not proceed via primitive rings), the case $C=8$ in [19], and the cases $C=4$ and $C=8$ in [18]. Nevertheless, they do not provide general lower bounds and they do not analyze the approximation ratio of the proposed algorithms. Therefore, the gaps between their solutions and the optimal ones are unknown.

In this work we focus on a bidirectional ring with symmetric shortest path routing, and on the all-toall case. We begin by formally stating the problem in terms of graph partitioning in Section 2. In Section 3 we provide lower bounds and compare them with those existing in the literature. The remainder of the article is devoted to finding families of solutions for certain values of $C$ and $N$. First we solve in Section 4 the case $C=1$. In Section 5 we study the case $C=2$, improving the general lower bound and providing a $\frac{34}{33}$-approximation. In Section 6 we tackle the case $C=3$, improving the lower bound when $N \equiv 3$ (mod 4) and giving optimal solutions when $N \equiv 0,1,4,5(\bmod 12)$. For all other values of $N$ we give asymptotically optimal solutions. In Section 7 we use design theory to provide optimal solutions when $C$ is of the form $k(k+1) / 2$, for some congruence classes of values of $N$. We also give improved lower bounds when $C$ is not of the form $k(k+1) / 2$. In Section 8 we compare unidirectional and bidirectional rings in terms of minimizing the cost. We conclude the article in Section 9.

\section{Statement of the Problem}

\subsection{Load constraint}

In a graph-theoretical approach, we are given an optical network represented by a directed graph $G$ on $N$ vertices (in many cases a symmetric one) - called the physical graph - for example a unidirectional ring $\vec{C}_{N}$ or a bidirectional symmetric ring $C_{N}^{*}$. We are given also a traffic (or instance) matrix, that is a family of connection requests represented by an arc-weighted multidigraph $I$ - called the logical or request graph - where the number of arcs from $i$ to $j$ corresponds to the number of requests from $i$ to $j$, and the weight of each arc corresponds to the amount of bandwidth used by each request. Here we suppose that there is exactly one request from $i$ to $j$ (all-to-all case) and that each request uses the same bandwidth. In that case $I=K_{N}^{*}$. We also suppose that the bandwidth used by any request is a fraction $1 / C$ of the available bandwidth of a wavelength. Said otherwise, each wavelength $\omega$ can carry on a given arc at most $C$ requests. This positive integer $C$ is called the grooming factor. For a wavelength $\omega$, we 
denote by $B_{\omega}$ the set of requests carried by $\omega$. Satisfying a request $r$ from $i$ to $j$ consists in finding a dipath $P(r)$ in $G$ and assigning it a wavelength $\omega$. Note that a wavelength $\omega$ is directed either clockwise or counterclockwise, so all the dipaths associated with requests in the same $B_{\omega}$ are directed in the same way.

For a subgraph $B_{\omega}$ of requests of $I$, we define the load of an $\operatorname{arc} e$ of $G, L\left(B_{\omega}, e\right)$, as the number of requests which are routed through $e$, that is

$$
L\left(B_{\omega}, e\right):=\left|\left\{P(r): r \in E\left(B_{\omega}\right), e \in P(r)\right\}\right| .
$$

Note that if $B_{\omega}$ is associated with a clockwise (resp. counterclockwise) wavelength $\omega$, only the clockwise (resp. counterclockwise) arcs of the ring are loaded by $B_{\omega}$. The constraint given by the grooming factor $C$ means that for each subgraph $B_{\omega}$ and each $\operatorname{arc} e, L\left(B_{\omega}, e\right)$ is at most $C$. In this article we focus on the bidirectional ring topology with all-to-all unitary requests. Therefore, our problem consists of finding a partition of $K_{N}^{*}$ into subdigraphs $B_{\omega}$ satisfying the load constraint for $C_{N}^{*}$ and such that the total number of vertices is minimized. We have two choices for routing a request $(i, j)$ : either clockwise or counterclockwise. Although there is no physical constraint imposing it, it is common for the operators to consider symmetric routings. That is, if the request $(i, j)$ is routed clockwise, then the request $(j, i)$ is routed counterclockwise. Furthermore it is also common for the sake of simplicity to use shortest path routing. Therefore we will restrict ourselves to symmetric shortest path routings. Let us see how the restrictions on the routing affect the solutions.

\subsection{Constraints on the routing}

In a ring $C_{N}^{*}$ with an odd number of vertices, shortest path routing implies symmetric routing. But in a ring with an even number of vertices this is not necessarily the case, as a request of the form $\left(i, i+\frac{N}{2}\right)$ can be routed via a shortest path in both directions. Consider for example $N=4$ and $C=2$. If we do not impose symmetric routing, we can have a solution consisting of the two subdigraphs $B_{\omega_{1}}$ with the requests $(0,1),(1,2),(2,3),(3,0),(0,2)$, and $(2,0)$ routed clockwise, and $B_{\omega_{2}}$ with the requests $(1,0)$, $(0,3),(3,2),(2,1),(1,3)$, and $(3,1)$ routed counterclockwise. Altogether we use 8 ADMs. Suppose now that we further impose symmetric routing, and assume without loss of generality that the requests $(0,2)$ and $(1,3)$ are routed clockwise. The best we can do for a $B_{\omega}$ with 4 vertices is to put 5 requests if $\omega$ is clockwise, namely $(0,1),(1,2),(2,3),(3,0)$, and at most one of $(0,2)$ and $(1,3)$. The other request out of $(0,2)$ and $(1,3)$ will need 2 ADMs, so we use a total of 12 ADMs. If we do not use any $B_{\omega}$ with 4 vertices, note that a subdigraph with 3 (resp. 2) vertices contains at most 3 requests (resp. 1 request). Therefore to route all the requests we need at least 12 ADMs.

Imposing shortest path routing might increase the number of ADMs of an optimal solution. Consider for example $N=3$ and $C=3$. With shortest path routing, we need two subdigraphs $B_{\omega_{1}}$ with the requests $(0,1),(1,2),(2,0)$ and $B_{\omega_{2}}$ with the requests $(1,0),(2,1),(0,2)$, for a total of 6 ADMs (each arc of $C_{3}^{*}$ is loaded once). Without the constraint of shortest path routing, we can do it with 3 ADMs, namely with 
all the requests routed clockwise. In that case, the requests $(1,0),(2,1)$, and $(0,2)$ are routed via dipaths of length 2 (for instance, the request $(1,0)$ uses the arcs $(1,2)$ and $(2,0)$ ). In that case the load of the arcs (in the clockwise direction) is 3.

We cannot always use shortest path routing and have a minimum load. Indeed, consider the case $C=1$ and a set of 3 requests $(i, j),(j, k)$, and $(k, i)$ forming a triangle. The subdigraph formed by the 3 requests routed in the same direction has load 1 , but there is no reason that the associated routes are shortest paths. For example, let $N=5$ and $(0,1),(1,2),(2,0)$ be the three mentioned requests, which we assume to be routed clockwise. If we want a valid solution, then the request $(2,0)$ is routed via the path $[2,3,4,0]$ of length 3 (and not 2). If we want to use shortest paths, then these three requests induce load 2 , hence they cannot fit together in the same wavelength. Summarizing, in this example either we use shortest paths and the load is 2 or we get a solution with load one but not using shortest paths.

\subsection{Symmetric shortest path routing}

In the sequel we will only consider symmetric shortest path routings. Besides being a common scenario in telecommunication networks, this assumption also simplifies the problem, as we can split it into two separate problems, half of the requests being routed clockwise and half counterclockwise. Each of these two subproblems can be viewed as a grooming problem where $G=\vec{C}_{N}$ (the unidirectional cycle) and $I=T_{N}$, where $T_{N}$ is a tournament on $N$ vertices, that is, a complete oriented graph (for each pair of vertices $\{i, j\}$ there is exactly one of the $\operatorname{arcs}(i, j)$ or $(j, i))$.

As we consider shortest path routing, for $N$ odd $T_{N}$ is unique. But for $N$ even we have two possibilities for the pairs of the form $\left\{i, i+\frac{N}{2}\right\}$ : either the $\operatorname{arc}\left(i, i+\frac{N}{2}\right)$ or $\left(i+\frac{N}{2}, i\right)$. So the choice of these arcs has to be made. We are now ready to state precisely our problem.

Traffic Grooming in Bidirectional WDM Ring Networks

Input: A unidirectional cycle $\vec{C}_{N}$ with vertices $0, \ldots, N-1$, a grooming factor $C$ and a digraph of requests consisting of the tournament $T_{N}$ with $\operatorname{arcs}(i, i+1)$ for $0 \leq i \leq N-1$ and $1 \leq q \leq \frac{N-1}{2}$, plus if $N$ is even $\frac{N}{2}$ arcs of the form $\left(i, i+\frac{N}{2}\right)$, where we cannot have both $\left(i, i+\frac{N}{2}\right)$ and $\left(i+\frac{N}{2}, i\right)$ (or said otherwise, for $N$ even we have one of the two $\operatorname{arcs}\left(i, i+\frac{N}{2}\right)$ or $\left(i+\frac{N}{2}, i\right)$ for $\left.0 \leq i \leq \frac{N}{2}-1\right)$. Output: A partition of $T_{N}$ into digraphs $B_{\omega}, 1 \leq \omega \leq W$, such that for each arc $e \in E\left(\vec{C}_{N}\right)$, $L\left(B_{\omega}, e\right) \leq C$.

Objective: Minimize $\sum_{\omega=1}^{W}\left|V\left(B_{\omega}\right)\right|$. The minimum will be denoted $A(C, N)$.

Note that for $N$ even we do not specify a particular orientation of the $\operatorname{arcs}$ of the form $\left(i, i+\frac{N}{2}\right)$.

Remark 2.1 Solutions to the original problem can be found by solving the above problem and using the solution for the counterclockwise requests by reversing the orientation of the arcs of $\vec{C}_{N}$ and $T_{N}$. Therefore, the total number of ADMs for the original problem - under the constraints of symmetric shortest path routing - is $2 A(C, N)$. 
Let us see an example for $N=5$ and $C=1$. Then the following three subdigraphs form a solution with 10 ADMs: one with arcs $(0,1),(1,3),(3,0)$, another with arcs $(1,2),(2,4),(4,1)$, and another with arcs $(0,2),(2,3),(3,4),(4,0)$. Thus, a solution for the bidirectional ring $C_{5}^{*}$ and $I=K_{5}^{*}$ needs 20 ADMs.

Let now $N=5$ and $C=2$. We can use the preceding solution or another one with also 10 ADMs with only two $\vec{C}_{5}$ 's with arcs $(0,2),(1,2),(2,3),(3,4),(4,5)$ and $(0,2),(2,4),(4,1),(1,3),(3,0)$, the second one inducing load 2. But we can do better, with only 8 ADMs, with one subdigraph with arcs $(1,3),(3,4),(4,1)$, and another one with arcs $(0,1),(1,2),(0,2),(2,3),(2,4),(3,0),(4,0)$. This latter partition is optimal. In that case, we need 16 ADMs for the bidirectional ring.

To tackle our problem we will use tools from design theory, similar to those used for the unidirectional ring and $I=K_{N}[7,8]$. In particular, it is helpful to use, for a given $C$, digraphs having a maximum ratio of the number of arcs to the number of vertices (see Section 3.2).

\subsection{Admissible digraphs}

Let $B_{\omega}=\left(V_{\omega}, E_{\omega}\right)$ be a digraph with $V_{\omega}=\left\{a_{0}, \ldots, a_{p-1}\right\}$ involved in a partition of the tournament $T_{N}$. Note that the edges of $B_{\omega}$ belong to $T_{N}$, so $\left(a_{i}, a_{j}\right) \in E_{\omega}$ if and only $d_{\overrightarrow{C_{N}}}\left(a_{i}, a_{j}\right) \leq \frac{N}{2}$, where $d_{\overrightarrow{C_{N}}}\left(a_{i}, a_{j}\right)$ is the distance between $a_{i}$ and $a_{j}$ in $\overrightarrow{C_{N}}$.

A digraph $B_{\omega}$ is said to be admissible if it satisfies the load constraint, that is, $L\left(B_{\omega}, e\right) \leq C$ for each arc $e \in E\left(\vec{C}_{N}\right)$. A partition of $T_{N}$ into admissible subdigraphs is called valid. As the paths associated with an arc of $B_{\omega}$ form a dipath (an interval) in $\vec{C}_{N}$, the load is exactly the same as if we consider $B_{\omega}$ embedded in a cycle $\vec{C}_{p}$ with vertex set $0,1, \ldots, p-1$. More precisely, we associate with $B_{\omega}$ the digraph $B_{\omega}^{p}$ having vertices $0,1, \ldots, p-1$ and with $(i, j) \in E\left(B_{\omega}^{p}\right)$ if and only if $\left(a_{i}, a_{j}\right) \in E\left(B_{\omega}\right)$. Hence, to compute the load we will consider digraphs with $p$ vertices and their load in the associated $\vec{C}_{p}$. Note that it can happen that $d_{\overrightarrow{C_{N}}}\left(a_{i}, a_{j}\right) \leq \frac{N}{2}$ but $d_{\overrightarrow{C_{p}}}(i, j)>\frac{p}{2}$, and vice versa.

Figure 1(a) illustrates a digraph $B_{\omega}$ that is admissible for $N=8$ and $C=2$, as it induces load 2 in $\vec{C}_{8}$. Its associated digraph $B_{\omega}^{4}$ is shown in Figure 1(b). Figure 1(c) shows a digraph $B_{\omega}^{\prime}$ which has also $B_{\omega}$ as associated digraph, but it is not admissible as $\left(a_{3}, a_{0}\right)$ is not an $\operatorname{arc}$ of $T_{8}$.

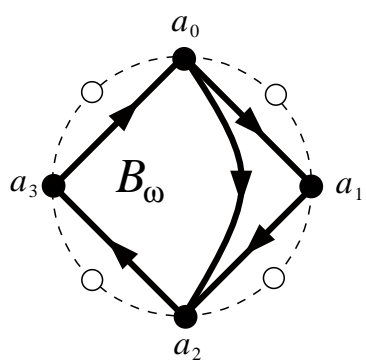

(a)

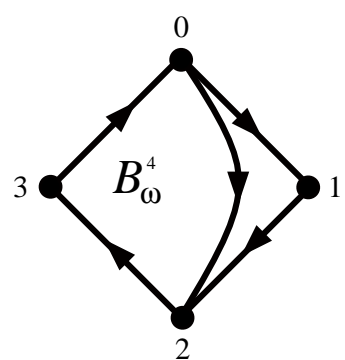

(b)

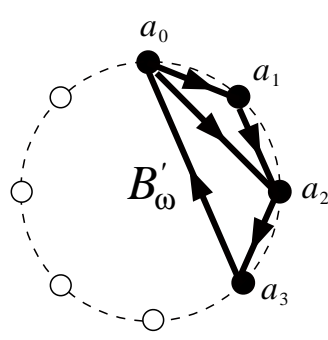

(c)

Figure 1: (a) Digraph $B_{\omega}$ admissible for $N=8$ and $C=2$; (b) Its associated digraph $B_{\omega}^{4}$; (c) Nonadmissible digraph $B_{\omega}^{\prime}$ that has also $B_{\omega}^{4}$ as associated digraph. 

$\operatorname{arc}\left(a_{1}, a_{4}\right)$ was in $\left.B_{\omega}\right)$.

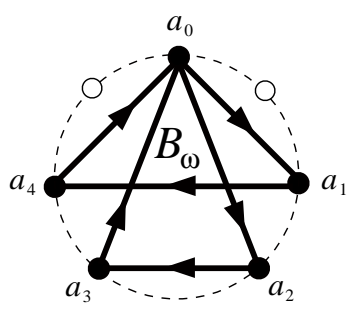

(a)

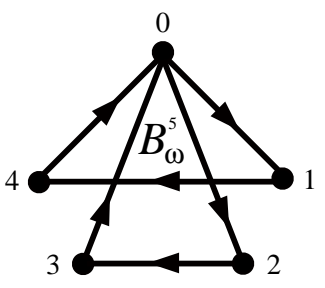

(b)

Figure 2: (a) Digraph $B_{\omega}$ admissible for $N=7$ and $C=2$; (b) Its associated digraph $B_{\omega}^{5}$.

Figure 2(a) shows an admissible digraph for $N=7$ and $C=2$. Its associated digraph $B_{\omega}^{5}$, which is depicted in Figure 2(b), induces load 2 but the arc $(1,4)$ is not routed via a shortest path (although the

Figure 2: (a) Digraph $B_{0}$ admissible for $N=7$ and $C=2 ;$ (b) Its associated digraph $B_{w}^{5}$.

In what follows we will compute the load in the associated digraph, but we will have to be careful that the arcs of $B_{\omega}$ are those of $T_{N}$, as pointed out by the above examples.

\section{Lower Bounds}

In this section we state general lower bounds on the number of ADMs used by any solution.

\subsection{Equations of the problem}

Given a valid solution of the problem, let $a_{p}$ denote the number of subgraphs of the partition with exactly $p$ nodes, let $A$ denote the total number of ADMs, let $W$ denote the number of subgraphs of the partition, and let $E_{\omega}$ be the set of arcs of $B_{\omega}$. Recall that here $I=T_{N}$, which has $\frac{N(N-1)}{2}$ arcs. The following equalities hold:

$$
\begin{aligned}
A & =\sum_{p=2}^{N} p a_{p} \\
\sum_{p=2}^{N} a_{p} & =W \\
\sum_{w=1}^{W}\left|E_{\omega}\right| & =\frac{N(N-1)}{2}
\end{aligned}
$$


Proposition 3.1 For $I=T_{N}$,

$$
W \geq\left\lceil\frac{N^{2}+\alpha}{8 C}\right\rceil, \text { where } \alpha=\left\{\begin{array}{cl}
-1, & \text { if } N \text { is odd } \\
4, & \text { if } N \equiv 2 \quad(\bmod 4) \\
8, & \text { if } N \equiv 0 \quad(\bmod 4)
\end{array}\right.
$$

Proof: The set of arcs of $T_{N}$ of the form $(i, i+q), 0 \leq q<\frac{N}{2}$, load each arc of the ring exactly $q$ times. So if $N$ is odd the load of any arc of the ring is $1+2+\cdots+\frac{N-1}{2}=\frac{N^{2}-1}{8}$.

If $N$ is even the load due to these arcs is $1+2+\cdots+\frac{N-2}{2}=\frac{N^{2}-2 N}{8}$. We have to add the load due to $\operatorname{arcs}$ of $T_{N}$ of the form $\left(i, i+\frac{N}{2}\right)$. As there are $\frac{N}{2}$ such arcs, the total load is $\frac{N^{2}}{4}$ and so one arc of the ring has load at least $\frac{N}{4}$.

If $N \equiv 2(\bmod 4)$ that gives a load at least $\left\lceil\frac{N}{4}\right\rceil=\frac{N+2}{4}$, so one arc has load at least $\frac{N^{2}-2 N}{8}+\frac{N+2}{4}=\frac{N^{2}+4}{8}$.

If $N \equiv 0(\bmod 4)$ the maximum load due to the $\operatorname{arcs}\left(i, i+\frac{N}{2}\right)$ is at least $\frac{N}{4}$, but in this case we can give a better bound. Indeed, suppose w.l.o.g. that we have the arc $\left(0, \frac{N}{2}\right)$, and let $j$ be the number of arcs starting in the interval $\left[1, \frac{N}{2}-1\right]$ of the form $\left(i, i+\frac{N}{2}\right)$ with $0<i<\frac{N}{2}$. The load of the $\operatorname{arc}\left(\frac{N}{2}-1, \frac{N}{2}\right)$ of the ring is then $j+1$. As there are $\frac{N}{2}-1-j$ arcs ending in the interval $\left[1, \frac{N}{2}-1\right]$, the load of the arc $(0,1)$ is $1+\frac{N}{2}-1-j$. Therefore the sum of the loads of the $\operatorname{arcs}(0,1)$ and $\left(\frac{N}{2}-1, \frac{N}{2}\right)$ is $\frac{N}{2}+1$, and so one of these $2 \operatorname{arcs}$ has load $\left\lceil\frac{N}{4}+\frac{1}{2}\right\rceil=\frac{N}{4}+1$. The total load of this arc is $\frac{N^{2}-2 N}{8}+\frac{N}{4}+1=\frac{N^{2}+8}{8}$.

As each subgraph can load one arc at most $C$ times, we obtain the lemma.

\subsection{The parameter $\gamma(C, p)$}

To obtain accurate lower bounds we need to bound the value of $\left|E_{\omega}\right|$ for a digraph with $\left|V_{\omega}\right|=p$ vertices, satisfying the load constraint (admissible digraph). As we discussed in the preceding section, we need only to consider the associated digraph embedded in $\vec{C}_{p}$. To this end, we introduce the following definitions.

Definition 3.1 Let $\gamma(C, p)$ be the maximum number of arcs of a digraph $H$ with $p$ vertices such that $L(H, e) \leq C$, for every arc e of $\vec{C}_{p}$.

\section{Definition 3.2}

$$
\rho(C)=\max _{p \geq 2}\left\{\frac{\gamma(C, p)}{p}\right\}
$$

In [33] the authors define two parameters which coincide with the parameters $\gamma(C, p)$ and $\rho(C)$ introduced above. In [33] the parameter $\rho(C)$ is called maximal ADM efficiency, and its value is determined, but no closed formula for $\gamma(C, p)$ is given in [33]. Here we give again the value of $\rho(C)$, using different tools, and give the exact value of $\gamma(C, p)$.

The next proposition shows that, in fact, the maximum number of requests we can groom is attained by taking those of minimum length. It is worth mentioning that this property is not true if the physical graph is a path, as shown with a counterexample in [3]. 
Proposition 3.2 Let $C=\frac{k(k+1)}{2}+r$, with $0 \leq r \leq k$. Then

$$
\gamma(C, p)=\left\{\begin{array}{cl}
\frac{p(p-1)}{2} & , \text { if } p \leq 2 k+1, \text { or } p=2 k+2 \text { and } r \geq \frac{k+2}{2} \\
k p+2 r-1 & , \text { if } p=2 k+2 \text { and } 1 \leq r<\frac{k+2}{2} \\
k p+\left\lfloor\frac{r p}{k+1}\right\rfloor & , \text { otherwise }
\end{array}\right.
$$

The graphs achieving $\gamma(C, p)$ are either the tournament $T_{p}$ if $p$ is small (namely, if $p \leq 2 k+1$ or $p=2 k+2$ and $r \geq \frac{k+2}{2}$ ), or subgraphs of a circulant digraph containing all the arcs of length $1,2, \ldots, k$, plus some arcs of length $k+1$ if $r>0$.

Proof: We distinguish three cases according to the value of $p$.

Case 1. If $p$ is small, that is such that the tournament $T_{p}$ loads each arc at most $C$ times, then $\gamma(C, p)=\frac{p(p-1)}{2}$. Let us now see for which values of $p$ this fact holds.

If $p$ is odd, the load of $T_{p}$ is $\frac{p^{2}-1}{8} \leq C$. The inequality $p^{2}-1 \leq 8 C$ implies $p^{2}-1 \leq 4 k(k+1)+8 r$, and is satisfied if $p \leq 2 k+1$, as $p^{2}-1 \leq 4 k(k+1)$.

If $p$ is even, the load of $T_{p}$ is $\frac{p^{2}}{8}+\frac{1+\delta}{2}$, where $\delta=1$ if $p \equiv 0(\bmod 4)$ (see proof of Proposition 3.1).

If $p \leq 2 k$, then $\frac{p^{2}+8}{8} \leq \frac{4 k^{2}+8}{8} \leq \frac{k(k+1)}{2} \leq C$.

For $p=2 k+2$, then $\frac{p^{2}}{8}+\frac{1+\delta}{2}=\frac{k^{2}}{2}+k+1+\frac{\delta}{2} \leq \frac{k^{2}+k}{2}+r=C$ if and only if $r \geq \frac{k+2+\delta}{2}$, with $\delta=1$ if $p \equiv 0(\bmod 4)$, that is, if $k$ is odd. Therefore, the condition is satisfied if $r \geq \frac{k+2}{2}$.

In the next two cases, we provide first a lower bound on $\gamma(C, p)$, and then we prove a matching upper bound.

Case 2. If $p=2 k+2$ and $1 \leq r<\frac{k+2}{2}$, a solution is obtained by taking all the arcs of length $1,2, \ldots, k\left(=\frac{p-2}{2}\right)$ - giving a load of $\frac{k(k+1)}{2}-$ plus $2 r-1$ arcs of length $\frac{p}{2}$. For example, we can take the $\operatorname{arcs}\left(i, i+\frac{p}{2}\right)$ for $i=0,2, \ldots, 2 r-2\left(<\frac{p}{2}\right)$ and the $\operatorname{arcs}\left(i, i-\frac{p}{2}\right)$ for $i=1,3, \ldots, 2 r-3$. The load due to these arcs is at most $r$. Therefore, in this case $\gamma(C, p) \geq k p+2 r-1$.

Case 3. If $p>2 k+2$ or $p=2 k+2$ and $r=0$, a solution is obtained by taking all the arcs of length $1,2, \ldots, k$ plus $\left\lfloor\frac{r p}{k+1}\right\rfloor$ arcs of length $k+1$, in such a way that the load due to these arcs is at most $C$, which is always possible (for example, if $p$ and $k+1$ are relatively prime, we take the requests $((k+1) i,(k+1)(i+1))$ for $0 \leq i \leq\left\lfloor\frac{r p}{k+1}\right\rfloor-1$, the indices being taken modulo $\left.p\right)$. Therefore, in this case

$$
\gamma(C, p) \geq k p+\left\lfloor\frac{r p}{k+1}\right\rfloor .
$$

Let us now turn to upper bounds. Suppose we have a solution with $\gamma \operatorname{arcs}, \gamma_{i}$ being of length $i$ on $\vec{C}_{p}$. 
As each arc of length $i$ loads $i$ arcs, and the total load of the $\operatorname{arcs}$ of $\vec{C}_{p}$ is at most $C p$, we have

$$
\begin{aligned}
C p & \geq \sum_{i=1}^{\infty} i \gamma_{i} \geq \sum_{i=1}^{k} i \gamma_{i}+(k+1)\left(\gamma-\sum_{i=1}^{k} \gamma_{i}\right) \\
& =\sum_{i=1}^{k} i p+(k+1)(\gamma-k p)+\sum_{i=1}^{k} \underbrace{(k+1-i)\left(p-\gamma_{i}\right)}_{\geq 0} \\
& \geq \frac{k(k+1)}{2} \cdot p+(k+1)(\gamma-k p) .
\end{aligned}
$$

Since $C p=\frac{k(k+1)}{2} \cdot p+r p$, we obtain $r p \geq(k+1)(\gamma-k p)$, and therefore

$$
\gamma(C, p) \leq k p+\frac{r p}{k+1} .
$$

Combining Relations (4) and (5), we get the result for Case 3. For Case 2, i.e., when $p=2 k+2$ and $1 \leq r<\frac{k+2}{2}$, Relation (5) yields $\gamma(C, p) \leq k p+2 r$. If we have equality, then necessarily $\gamma_{i}=p$ for $i=1, \ldots, k$, so we have all arcs of length at most $k$. However, the $2 r \operatorname{arcs}$ of length at least $k+1$ induce a load at least $r+1$ on some arc of $\vec{C}_{p}$, so the total load would be strictly greater than $C$. Therefore, we have at most $\gamma(C, p) \leq k p+2 r-1$, which gives the result.

Proposition 3.3 Let $C=k(k+1) / 2+r$, with $0 \leq r \leq k$. Then

$$
\rho(C)=k+\frac{r}{k+1}
$$

Proof: In Case 1 of the proof of Proposition 3.2, $\rho(C) \leq \frac{p-1}{2}$. If $p \leq 2 k+1, \rho(C) \leq k$. If $p=2 k+2$ and $r \geq \frac{k+2}{2}, \rho(C)=k+\frac{1}{2}<k+\frac{r}{k+1}$. Otherwise, by Relation (5),

$$
\rho(C) \leq \frac{k p+\frac{r p}{k+1}}{p}=k+\frac{r}{k+1},
$$

where $C=\frac{k(k+1)}{2}+r$, with $0 \leq r \leq k$. So, in all cases, $\rho(C) \leq k+\frac{r}{k+1}$. Note that when $p$ is a multiple of $k+1$, Relation (4) implies that $\gamma(C, p) \geq k p+\frac{r p}{k+1}$, and therefore $\rho(C) \geq k+\frac{r}{k+1}$. The result follows.

Note that in [33] the following formula is given, equivalent to Equation (6):

$$
\rho(C)=\frac{C}{k+1}+\frac{k}{2} .
$$

Table 1 shows the parameter $\gamma(C, p)$ for small values of $C$ and $p$, as well as the parameter $\rho(C)$.

\subsection{General lower bounds}

By Propositions 3.1 and 3.2, Equations (1), (2), and (3) become 


\begin{tabular}{|c||c|c|c|c|c|c|c|c|c|c|c|c|c|c|c||c|}
\hline$p$ & 2 & 3 & 4 & 5 & 6 & 7 & 8 & 9 & 10 & 11 & 12 & 13 & 14 & 15 & 16 & $\rho(C)$ \\
\hline \hline$C=1$ & 1 & $\mathbf{3}$ & $\mathbf{4}$ & $\mathbf{5}$ & $\mathbf{6}$ & $\mathbf{7}$ & $\mathbf{8}$ & $\mathbf{9}$ & $\mathbf{1 0}$ & $\mathbf{1 1}$ & $\mathbf{1 2}$ & $\mathbf{1 3}$ & $\mathbf{1 4}$ & $\mathbf{1 5}$ & $\mathbf{1 6}$ & 1 \\
\hline$C=2$ & 1 & 3 & 5 & 7 & 9 & 10 & $\mathbf{1 2}$ & 13 & $\mathbf{1 5}$ & 16 & $\mathbf{1 8}$ & 19 & $\mathbf{2 1}$ & 22 & $\mathbf{2 4}$ & $3 / 2$ \\
\hline$C=3$ & 1 & 3 & 6 & $\mathbf{1 0}$ & $\mathbf{1 2}$ & $\mathbf{1 4}$ & $\mathbf{1 6}$ & $\mathbf{1 8}$ & $\mathbf{2 0}$ & $\mathbf{2 2}$ & $\mathbf{2 4}$ & $\mathbf{2 6}$ & $\mathbf{2 8}$ & $\mathbf{3 0}$ & $\mathbf{3 2}$ & 2 \\
\hline$C=4$ & 1 & 3 & 6 & 10 & 13 & 16 & 18 & $\mathbf{2 1}$ & 23 & 25 & $\mathbf{2 8}$ & 30 & 32 & $\mathbf{3 5}$ & 37 & $7 / 3$ \\
\hline$C=5$ & 1 & 3 & 6 & 10 & 15 & 18 & 21 & $\mathbf{2 4}$ & 26 & 29 & $\mathbf{3 2}$ & 34 & 37 & $\mathbf{4 0}$ & 42 & $8 / 3$ \\
\hline$C=6$ & 1 & 3 & 6 & 10 & 15 & $\mathbf{2 1}$ & $\mathbf{2 4}$ & $\mathbf{2 7}$ & $\mathbf{3 0}$ & $\mathbf{3 3}$ & $\mathbf{3 6}$ & $\mathbf{3 9}$ & $\mathbf{4 2}$ & $\mathbf{4 5}$ & $\mathbf{4 8}$ & 3 \\
\hline$C=7$ & 1 & 3 & 6 & 10 & 15 & 21 & 25 & 29 & 32 & 35 & $\mathbf{3 9}$ & 42 & 45 & 48 & $\mathbf{5 2}$ & $13 / 4$ \\
\hline$C=8$ & 1 & 3 & 6 & 10 & 15 & 21 & 27 & 31 & 35 & 38 & $\mathbf{4 2}$ & 45 & 49 & 52 & $\mathbf{5 6}$ & $14 / 4$ \\
\hline$C=9$ & 1 & 3 & 6 & 10 & 15 & 21 & 28 & 33 & 37 & 41 & $\mathbf{4 5}$ & 48 & 52 & 56 & $\mathbf{6 0}$ & $15 / 4$ \\
\hline$C=10$ & 1 & 3 & 6 & 10 & 15 & 21 & 28 & $\mathbf{3 6}$ & $\mathbf{4 0}$ & $\mathbf{4 4}$ & $\mathbf{4 8}$ & $\mathbf{5 2}$ & $\mathbf{5 6}$ & $\mathbf{6 0}$ & $\mathbf{6 4}$ & 4 \\
\hline
\end{tabular}

Table 1: The parameter $\gamma(C, p)$ for some values of $C$ and $p$, as well as $\rho(C)$. The bold values achieve $\rho(C)$.

$$
\begin{aligned}
A & =\sum_{p=2}^{N} p a_{p} \\
\sum_{p=2}^{N} a_{p} & \geq\left\lceil\frac{N^{2}+\alpha}{8 C}\right\rceil, \text { where } \alpha=\left\{\begin{array}{ccc}
-1 & , \text { if } N \text { is odd } \\
4 & , \text { if } N \equiv 2 \quad(\bmod 4) \\
8 & , \text { if } N \equiv 0 \quad(\bmod 4)
\end{array}\right. \\
\sum_{p=2}^{N} a_{p} \gamma(C, p) & \geq \frac{N(N-1)}{2}
\end{aligned}
$$

Theorem 3.1 (General lower bound) Let $C=\frac{k(k+1)}{2}+r$, with $0 \leq r \leq k$. The number of ADMs required in a bidirectional ring with $N$ nodes and grooming factor $C$ satisfies

$$
A(C, N) \geq\left\lceil\frac{N(N-1)}{2 \cdot \rho(C)}\right\rceil=\left\lceil\frac{N(N-1)}{2} \frac{k+1}{k(k+1)+r}\right\rceil .
$$

Proof: Using Equation (9) and Relation (11), and the definition of $\rho(C)$, we get that the number $A$ of ADMs used by any solution satisfies

$$
\frac{N(N-1)}{2} \leq \sum_{p=2}^{N} a_{p} \cdot \gamma(C, p)=\sum_{p=2}^{N} p \cdot a_{p} \cdot \rho(C)=\rho(C) \cdot A .
$$


From the above relation and using Relation (7), we get

$$
A \geq\left\lceil\frac{N(N-1)}{2 \cdot \rho(C)}\right\rceil=\left\lceil\frac{N(N-1)}{2} \frac{k+1}{k(k+1)+r}\right\rceil .
$$

\section{Theorem 4.1} request graph. routing, Table 1).

To achieve the lower bound of Theorem 3.1, the only possibility is to use graphs on $p$ vertices with $\gamma(C, p)$ arcs. The bold values in Table 1 achieve $\rho(C)$, and therefore the subgraphs corresponding to those values (which exist by Proposition 3.2) are good candidates to construct an optimal partition of the

Comparison with existing lower bounds. In [14] the RING TrafFic Grooming problem in the bidirectional ring is studied. The authors state a lower bound regardless of routing for a general set of requests. In the particular case of uniform traffic, they get a lower bound of $\frac{N^{2}-1}{4 \sqrt{2 C}}$ (see [14, Theorem 1, page 198]). They indicate in their article that they can improve this bound by a factor of 2 for all-to-all uniform unitary traffic. We thank T. Chow and P. Lin for sending us the proof of the following theorem, which is only announced in [14].

Theorem 3.2 $([13,14])$ If a traffic instance of ring grooming is uniform and unitary, then, regardless of

$$
A(C, N) \geq \frac{1}{2 \sqrt{C}} \sqrt{\frac{N^{2}(N-1)^{2}}{2}-N(N-1)} .
$$

The lower bound we obtained in Theorem 3.1 is greater than the bound of Theorem 3.2, but it should be observed that we restrict ourselves to shortest path symmetric routing. Our bound is $\frac{N(N-1)}{2 \rho(C)}$ and the lower bound of Theorem 3.2 is less than $\frac{N(N-1)}{2 \sqrt{2 C}}$. The fact that our bound is better follows from the fact that $\rho(C)<\sqrt{2 C}$. Indeed,

$$
\rho^{2}(C) \leq\left(k+\frac{r}{k+1}\right)^{2}=k^{2}+\frac{2 k r}{k+1}+\frac{r^{2}}{(k+1)^{2}}<k^{2}+2 r+1<k^{2}+k+2 r=2 C .
$$

\section{Case $C=1$}

For $C=1$, by Proposition $3.2 \gamma(1, p)=p$ if $p \geq 2$. Furthermore, all the directed cycles achieve $\rho(1)$ (see

$$
A(1, N)=\left\{\begin{array}{cl}
\frac{N(N-1)}{2} & , \text { if } N \text { is odd } \\
\frac{N^{2}}{2} & , \text { if } N \text { is even }
\end{array}\right.
$$


Proof: For $C=1$, the only possible subgraphs involved in the partition of the edges of $T_{N}$ are cycles and paths. If only cycles are used, the total number of ADMs is $\frac{N(N-1)}{2}$, which equals the lower bound of Theorem 3.1. Each path involved in the partition adds one unit of cost with respect to $\frac{N(N-1)}{2}$.

If $N=2 q+1$ is odd, by [10, Theorem 3.3] we know that the arcs of $T_{N}$ can be covered with $q \vec{C}_{3}$ 's and $\frac{q(q-1)}{2} \vec{C}_{4}$ 's. The total number of vertices of this construction is $3 q+2 q(q-1)=q(2 q+1)=\frac{N(N-1)}{2}$.

If $N$ is even, each vertex must appear with odd degree in at least one subgraph, so the number of paths in any construction is at least $N / 2$. Therefore, the lower bound becomes $\frac{N(N-1)}{2}+\frac{N}{2}=\frac{N^{2}}{2}$. By [10, Theorem 3.4] the arcs of $T_{N}$ can be covered with

- $4 \vec{C}_{3}$ 's and $2 q^{2}-3 \vec{C}_{4}$ 's, if $N=4 q$ with $q>1$;

- $2 \vec{C}_{3}$ 's and $2 q^{2}+2 q-1 \vec{C}_{4}$ 's, if $N=4 q+2$.

For $N=4$, we cover $T_{4}$ with a $\vec{C}_{4}$ and two arcs. Note that in these constructions, some arcs are covered more than once. In both cases, the total number of vertices of the construction is $\frac{N^{2}}{2}$, hence the lower bound is attained.

Finally, one can check that in the constructions of [10], the length of the arcs involved in the covering of $T_{N}$ is in all cases bounded above by $\left\lfloor\frac{N}{2}\right\rfloor$, and therefore all the cycles induce load 1.

Remark 4.1 For the original problem with $G=C_{N}^{*}$ and $I=K_{N}^{*}$, if we apply Theorem 4.1 we get in the case $N$ even a value of $N^{2} A D M S$; but if we delete the constraint of symmetric routings we get a value of $N(N-1) / 2$ by using [10, Theorems 4.1 and 4.2] (however these constructions use many $K_{2}$ 's).

\section{Case $C=2$}

When $C=2$ the general lower bound of Theorem 3.1 gives $A(2, N) \geq \frac{N(N-1)}{3}$. We first improve this bound in Section 5.1, and then give solutions with a good approximation ratio in Section 5.2.

\subsection{Improved lower bounds}

For $C=2$, by Proposition $3.2 \gamma(2,2)=1, \gamma(2,3)=3, \gamma(2,4)=5$ (note that $\gamma(2,4)=6$ if the routing is not restricted to be symmetric), and $\gamma(2, p)=\left\lfloor\frac{3 p}{2}\right\rfloor$ for $p \geq 5$. The optimal solutions for $p \geq 4$ even consist of the $p$ arcs of length $1(i, i+1)$ for $0 \leq i \leq p-1$, plus the $p / 2 \operatorname{arcs}$ of length $2(2 i, 2 i+2)$ for $0 \leq i \leq p / 2-1$ (in fact, triangles sharing a vertex; see Figure 3 for $p=6$ ). For $p$ odd we have two classes of optimal graphs (see Figure 3 for $p=5$ ).
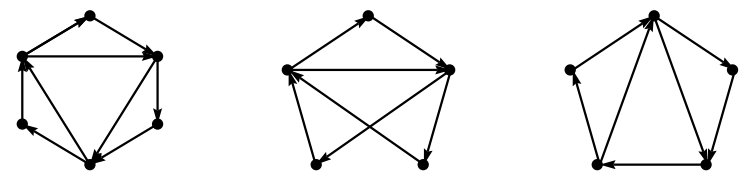

Figure 3: Some admissible digraphs for $C=2$. 
Relation (11) becomes in the case $C=2$

$$
\sum_{p=2}^{N} a_{p} \gamma(2, p)=a_{2}+3 a_{3}+5 a_{4}+7 a_{5}+9 a_{6}+10 a_{7}+12 a_{8}+\cdots \geq \frac{N(N-1)}{2} .
$$

Therefore,

$$
\begin{aligned}
A & =\sum_{p=2}^{N} p a_{p} \geq \frac{2}{3} \sum_{p=2}^{N} a_{p} \gamma(2, p)+\frac{4}{3} a_{2}+a_{3}+\frac{2}{3} a_{4}+\frac{1}{3}\left(a_{5}+a_{7}+a_{9}+\cdots\right) \\
& \geq \frac{N(N-1)}{3}+\frac{4}{3} a_{2}+a_{3}+\frac{2}{3} a_{4}+\frac{1}{3}\left(a_{5}+a_{7}+a_{9}+\cdots\right) .
\end{aligned}
$$

We can already see that the bound $\frac{N(N-1)}{3}$ cannot be attained. Indeed, to reach it we need to use only graphs with $6,8,10, \ldots$ vertices. But the number of graphs $W$ satisfies, by Proposition $3.1, W \geq \frac{N^{2}-1}{16}$, so $A \geq 6 \frac{N^{2}-1}{16}>\frac{N(N-1)}{3}$.

The following proposition gives a lower bound of order $\frac{11}{32} N(N-1)$. Note that $11 / 32>11 / 33=1 / 3$.

Proposition 5.1 (Tighter lower bound for $C=2$ )

$$
A(2, N) \geq\left\lceil\frac{11 N^{2}-8 N-3}{32}\right\rceil=\left\lceil\frac{11}{16} \frac{N(N-1)}{2}+\frac{3 N-3}{32}\right\rceil .
$$

Proof: We can write $A \geq 6\left(W-a_{2}-a_{3}-a_{4}-a_{5}\right)+2 a_{2}+3 a_{3}+4 a_{4}+5 a_{5}$, that is,

$$
A \geq 6 W-\left(4 a_{2}+3 a_{3}+2 a_{4}+a_{5}\right) .
$$

From Relations (13) and (14) we get

$$
3 A \geq N(N-1)+\left(4 a_{2}+3 a_{3}+2 a_{4}+a_{5}\right)
$$

Summing Relations (16) and (17) gives

$$
4 A \geq 6 W+N(N-1)
$$

By Proposition 3.1, we have that

$$
W \geq \frac{N(N-1)}{16}+\frac{N+\alpha}{16} .
$$

Combining Relations (18) and (19) and using $\alpha \geq-1$ yields

$$
A \geq \frac{11 N(N-1)}{32}+\frac{3 N}{32}+\frac{3 \alpha}{32} \geq \frac{11 N^{2}-8 N-3}{32} .
$$




\subsection{Upper bounds}

In this section we build families of solutions for $C=2$. We conjecture that there exists a decomposition using $A$ vertices with ratio $\frac{A}{\frac{N(N-1)}{2}}$ of order $\frac{11}{16}$, which would be optimal by Proposition 5.1. For that, we should find some (multipartite) graphs achieving this ratio. A candidate is $K_{4,4,4}$, which has 48 edges. Unfortunately, we have not been able to cover it with 33 vertices (which would achieve the optimal ratio) but only with 34 , giving a 34/33-approximation.

For the sake of the presentation, we first present a simple 12/11-approximation inspired from a construction of [10].

\subsubsection{A 12/11-approximation}

This construction is defined recursively. Suppose we have a solution for $N$ vertices using $A_{N}$ ADMs, with $N=2 p$ or $N=2 p+1$. Let the vertex set be labeled $0_{A}<1_{A}<\cdots<(p-1)_{A}<0_{B}<1_{B}<\cdots<(p-1)_{B}$, plus $\infty$ is $N$ is odd. For $N+2$, we add two vertices $x_{A}$ and $x_{B}$ with the order $x_{A}<0_{A}<1_{A}<\cdots<$ $(p-1)_{A}<x_{B}<0_{B}<1_{B}<\cdots<(p-1)_{B}<\infty$. We use as subdigraphs those of the solution for $N$ plus the $\lfloor p / 2\rfloor$ digraphs on the 6 vertices $x_{A}, i_{A},(i+\lfloor p / 2\rfloor)_{A}, x_{B}, i_{B},(i+\lfloor p / 2\rfloor)_{B}$ and the $8 \operatorname{arcs}\left(x_{A}, i_{A}\right)$, $\left(x_{A},(i+\lfloor p / 2\rfloor)_{A}\right),\left(i_{A}, x_{B}\right),\left((i+\lfloor p / 2\rfloor)_{A}, x_{B}\right),\left(x_{B}, i_{B}\right),\left(x_{B},\left(i+\lfloor p / 2\rfloor_{B}\right),\left(i_{B}, x_{A}\right),\left((i+\lfloor p / 2\rfloor)_{B}, x_{A}\right)\right.$, for $0 \leq i \leq\lfloor p / 2\rfloor-1$.

If $N=2 p$ with $p$ even, there remains uncovered the $\operatorname{arc}\left(x_{A}, x_{B}\right)$.

If $N=2 p+1$ with $p$ even, there remain the $3 \operatorname{arcs}\left(x_{A}, x_{B}\right),\left(x_{B}, \infty\right)$, and $\left(\infty, x_{A}\right)$, which we cover with the circuit $\left(x_{A}, x_{B}, \infty\right)$.

If $N=2 p$ with $p$ odd, there remain the $5 \operatorname{arcs}\left(x_{A},(p-1)_{A}\right),\left((p-1)_{A}, x_{B}\right),\left(x_{B},(p-1)_{B}\right),\left((p-1)_{B}, x_{A}\right)$, and $\left(x_{A}, x_{B}\right)$, which we cover with a digraph on 4 vertices containing all of them.

Finally, if $N=2 p+1$ with $p$ odd, there remain the $7 \operatorname{arcs}\left(x_{A},(p-1)_{A}\right),\left((p-1)_{A}, x_{B}\right),\left(x_{B},(p-\right.$ $\left.1)_{B}\right),\left((p-1)_{B}, x_{A}\right),\left(x_{A}, x_{B}\right),\left(x_{B}, \infty\right)$, and $\left(\infty, x_{A}\right)$, which we cover with a digraph on 5 vertices containing all of them.

One can check that, in all cases, the arcs $(u, v)$ considered satisfy $d_{\vec{C}_{n}}(u, v) \leq N / 2$.

To compute the number of ADMs of this construction, we have the recurrence relations $A_{4 q+2}=$ $A_{4 q}+6 q+2, A_{4 q+4}=A_{4 q+2}+6 q+4, A_{4 q+3}=A_{4 q+1}+6 q+3$, and $A_{4 q+5}=A_{4 q+3}+6 q+5$. Starting with $A_{2}=2$ or $A_{4}=6$ (obtained with the partition with the digraph on 4 vertices formed by the $C_{4}(0,1,2,3)$ plus the arc $(0,2)$ and the digraph on 2 vertices $(1,3))$ and $A_{3}=3$ or $A_{5}=8$ (obtained with the partition of $T_{5}$ using the first digraph on 5 vertices of Figure 3 and the remaining $T_{3}$ ), we get $A_{4 q}=6 q^{2}=\frac{6 N^{2}}{16}$, $A_{4 q+2}=6 q^{2}+6 q+2=\frac{6 N^{2}+8}{16}, A_{4 q+1}=6 q^{2}+2 q=\frac{6 N^{2}-4 N-2}{16}$, and $A_{4 q+3}=6 q^{2}+8 q+3=\frac{6 N^{2}-4 N+6}{16}$.

In all cases, the number of ADMs is of order $\frac{6}{8} \frac{N(N-1)}{2}$, so asymptotically the ratio between the number of ADMs of this construction and the lower bound of Proposition 5.1 tends to $\frac{6}{8} \frac{16}{11}=\frac{12}{11}$. 


\subsubsection{A 34/33-approximation}

It will be useful to use the notation $G_{5}$ and $G_{6}$ to refer to the digraphs depicted in Figure 4 . The key idea of this construction is that an oriented tripartite graph $K_{4,4,4}$ can be partitioned into admissible subdigraphs for $C=2$ using 34 vertices overall, as follows.

Let the tripartition classes of the $K_{4,4,4}$ be $\left\{1_{A}, 1_{B}, 1_{C}, 1_{D}\right\},\left\{2_{A}, 2_{B}, 2_{C}, 2_{D}\right\},\left\{3_{A}, 3_{B}, 3_{C}, 3_{D}\right\}$, and let the vertices be ordered in the ring $1_{A}<2_{A}<3_{A}<1_{B}<2_{B}<3_{B}<1_{C}<2_{C}<3_{C}<1_{D}<$ $2_{D}<3_{D}$. The arcs of an oriented $K_{4,4,4}$ can be partitioned into $4 G_{6}$ 's with $\left\{x_{1}, x_{2}, x_{3}, x_{4}, x_{5}, x_{6}\right\}=$ $\left\{1_{A}, 2_{A}, 3_{B}, 1_{C}, 2_{C}, 3_{D}\right\},\left\{1_{B}, 2_{B}, 3_{B}, 1_{D}, 2_{D}, 3_{D}\right\},\left\{1_{B}, 2_{C}, 3_{C}, 1_{D}, 2_{A}, 3_{A}\right\}$, and $\left\{1_{A}, 3_{A}, 2_{B}, 1_{C}, 3_{C}, 2_{D}\right\}$, plus $2 G_{5}$ 's with $\left\{x_{1}, x_{2}, x_{3}, x_{4}, x_{5}\right\}=\left\{3_{A}, 1_{C}, 2_{C}, 1_{D}, 2_{D}\right\}$ and $\left\{3_{D}, 2_{A}, 2_{B}, 1_{D}, 1_{C}\right\}$ (see Figure 4). The total number of vertices of this partition is 34 .
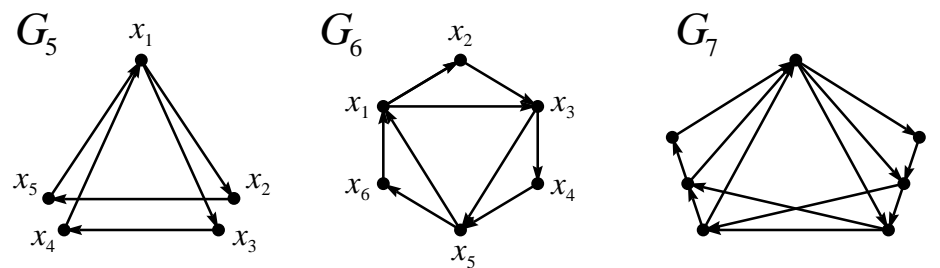

Figure 4: Digraphs $G_{5}$ and $G_{6}$ used in the 34/33-approximation for $C=2$, and digraph $G_{7}$ suitable for $C=3$ referred to in the proof of Proposition 6.2.

We are now ready to explain the construction. We take an integer $p \equiv 1$ or $3(\bmod 6)$, hence $K_{p}$ can be partitioned into triangles. We replace each vertex $i$ of $K_{p}$ with 4 vertices $i_{A}, i_{B}, i_{C}, i_{D}$, and order the vertices $1_{A}<\cdots<p_{A}<1_{B}<2_{B}<\cdots<p_{B}<1_{C}<\cdots<p_{C}<1_{D}<\cdots<p_{D}$. To a triple $\{i, j, k\}$ corresponding to a triangle of $K_{p}$, with $i<j<k$, we associate the decomposition described above of the $K_{4,4,4}$ on vertices $\left\{\ell_{A}, \ell_{B}, \ell_{C}, \ell_{D}: \ell=i, j, k\right\}$. In this way, $K_{p \times 4}$ can be partitioned into $\frac{p(p-1)}{6} K_{4,4,4}$ 's, or equivalently into $\frac{p(p-1)}{6} \cdot 4 G_{6}$ 's and $\frac{p(p-1)}{6} \cdot 2 G_{5}$ 's. Overall, we use $\frac{34 p(p-1)}{6}$ vertices. Each of the subdigraphs of this partition is admissible, as the distance in the ring between the endpoints of an arc is strictly smaller than $2 p$.

To partition an oriented $K_{4 p}$, there remain only the $K_{4}$ 's induced inside each class of the $K_{p \times 4}$. As $A(2,4)=6$, we use $6 p$ vertices to cover all the $K_{4}$ 's.

Therefore, if $p \equiv 1$ or $3(\bmod 6)$, an oriented $K_{4 p}$ can be partitioned using $6 p+\frac{34 p(p-1)}{6}=\frac{34 p^{2}+2 p}{6}=$ $\frac{34 N^{2}+8 N}{96}$ vertices. To decompose $K_{4 p+1}$, we add a vertex $\infty$, and we partition the $p K_{5}$ 's using 8 vertices for each one of them. Overall, we use $8 p+\frac{34 p(p-1)}{6}=\frac{34 p^{2}+14 p}{6}=\frac{34 N^{2}-12 N-24}{96}$ vertices.

If $p \not \equiv 1$ or $3(\bmod 6)$, we introduce dummy vertices to get $p^{\prime} \equiv 1$ or $3(\bmod 6)$, we do the construction described above, and then we remove the dummy edges and vertices. It is clear that these dummy vertices add $O(N)$ vertices to the construction, hence the coefficient of the term $N^{2}$ remains the same.

Since $\frac{33 N^{2}-24 N-9}{96}$ is a lower bound by Proposition 5.1, we get the following result.

Proposition 5.2 The above construction approximates $A(2, N)$ within a factor $34 / 33$. 


\section{Case $C=3$}

We first provide improved lower bounds for some congruence classes in Section 6.1 and then we provide constructions in Section 6.2, which are either optimal or asymptotically optimal.

\subsection{Improved lower bounds}

In this case (see Table 1) we have $\gamma(3,2)=1, \gamma(3,3)=3, \gamma(3,4)=6$, and $\gamma(3, p)=2 p$ for $p \geq 5$, so $\rho(3)=2$. Therefore, by Theorem 3.1, we get

Proposition 6.1 $A(3, N) \geq \frac{N(N-1)}{4}$.

By Relations (9) and (11) we have

$$
\begin{aligned}
2 A & =\sum_{p=2}^{N} 2 p a_{p}=4 a_{2}+6 a_{3}+8 a_{4}+\sum_{p=5}^{N} 2 p a_{p} \\
\frac{N(N-1)}{2} & \leq \sum_{p=2}^{N} a_{p} \gamma(3, p)=a_{2}+3 a_{3}+6 a_{4}+\sum_{p=5}^{N} 2 p a_{p}
\end{aligned}
$$

So

$$
A \geq \frac{N(N-1)}{4}+\frac{3}{2} a_{2}+\frac{3}{2} a_{3}+a_{4} .
$$

Therefore, if the lower bound is attained, then necessarily $a_{2}=a_{3}=a_{4}=0$. We will see in Section 6.2 that this is the case for $N \equiv 1$ or $5(\bmod 12)$, using optimal digraphs on 5 vertices (namely $\left.T_{5}\right)$ and on 6 vertices (namely $\vec{K}_{2,2,2}$, see Figure 5). Optimal graphs are obtained by using arcs of length 1 and 2, so the degree of any vertex in an optimal subdigraph is 4 . That is possible only if the total degree of a vertex, namely $N-1$, is a multiple of 4 . Otherwise, the following proposition shows that the lower bound of Proposition 6.1 cannot be attained.

\section{Proposition 6.2}

If $N \equiv 3(\bmod 4), \quad A(3, N) \geq \frac{N(N-1)}{4}+\frac{N}{6}=\frac{3 N^{2}-N}{12}$.

If $N \equiv 0(\bmod 2), \quad A(3, N) \geq \frac{N(N-1)}{4}+\frac{N}{4}=\frac{N^{2}}{4}$.

Proof: We use the following observation: If a vertex $x$ has out-degree 3 (resp. in-degree 3 ) in a digraph $B_{\omega}$, then its nearest out-neighbor $A_{x}^{+}$(resp. in-neighbor $A_{x}^{-}$) has in-degree 1 and out-degree at most 1 (resp. out-degree 1 and in-degree at most 1). Indeed, suppose $x$ has out-degree 3 , and let $A_{x}^{+}, B_{x}^{+}, C_{x}^{+}$be the out-neighbors of $x$. Then the load of the $\operatorname{arc}$ entering $A_{x}^{+}$is already 3 , so $A_{x}^{+}$has no other in-neighbor than $x$. The load of the arc leaving $A_{x}^{+}$is already 2 , so $A_{x}^{+}$has at most 1 out-neighbor $y$. If $y$ has 2 or more in-neighbors, then $A_{x}^{+}$is not its nearest one. Hence, to each vertex $x$ of out-degree 3 (resp. in-degree 3 ) is associated a distinct vertex $A_{x}^{+}$(resp. $A_{x}^{-}$) of degree at most 2 . 
Consider the digraphs in which a given vertex $x$ appears. Let $\alpha_{i}^{x}$ be the number of times $x$ appears with degree $i$, and let $\alpha_{i}=\sum_{x} \alpha_{i}^{x}$. Vertex $x$ appears in $\sum_{i} \alpha_{i}^{x}$ digraphs, so

$$
A=\sum_{x} \sum_{i} \alpha_{i}^{x}=\sum_{i} \alpha_{i}
$$

As each vertex has degree $N-1, N-1=\sum_{i} i \cdot \alpha_{i}^{x}$, and so

$$
N(N-1)=\sum_{x} \sum_{i} i \cdot \alpha_{i}^{x}=\sum_{i} i \cdot \alpha_{i}
$$

Due to the load constraint, a vertex has out-degree (resp. in-degree) at most 3 in all the digraphs in which it appears. Therefore, its degree is at most 6 , that is, $\alpha_{i}=0$ for $i \geq 7$. Furthermore, by the above observation if a vertex has degree 6 (resp. 5), to this vertex are associated 2 vertices (resp. 1 vertex) of degree at most 2 , and all these vertices are distinct, so

$$
\alpha_{1}+\alpha_{2} \geq 2 \alpha_{6}+\alpha_{5}
$$

Combining Equations (20) and (21) we get

$$
4 A=N(N-1)+3 \alpha_{1}+2 \alpha_{2}+\alpha_{3}-\alpha_{5}-2 \alpha_{6}
$$

We distinguish two cases: $N$ even or $N=4 t+3$.

If $N$ is even, $N-1$ is odd and each vertex must appear at least in one $B_{\omega}$ with odd degree, so

$$
\alpha_{1}+\alpha_{3}+\alpha_{5} \geq N
$$

Using Relation (22) multiplied by 2 in Relation (23) we get $4 A \geq N(N-1)+\alpha_{1}+\alpha_{3}+\alpha_{5}+2 \alpha_{6}$, so by Relation (24), $4 A \geq N(N-1)+N$, as claimed. Note that to obtain equality we need $\alpha_{6}=0, \alpha_{1}+\alpha_{2}=\alpha_{5}$, and $\alpha_{1}+\alpha_{3}+\alpha_{5}=N$.

If $N=4 t+3$, the degree of each vertex satisfies $N-1 \equiv 2(\bmod 4)$, so no vertex can appear with degree 4 in all the digraphs. Each vertex must appear either at least once with degree 6 or 2, or at least twice with odd degree (for example, 5 and 5, 3 and 3,1 and 1, or 5 and 1), so

$$
\alpha_{2}+\alpha_{6}+\frac{1}{2}\left(\alpha_{1}+\alpha_{3}+\alpha_{5}\right) \geq N
$$

Equation (23) can be rewritten as

$$
4 A=N(N-1)+\frac{2}{3}\left(\alpha_{2}+\alpha_{6}+\frac{1}{2}\left(\alpha_{1}+\alpha_{3}+\alpha_{5}\right)\right)+\frac{4}{3}\left(\alpha_{2}+\alpha_{1}-2 \alpha_{6}-\alpha_{5}\right)+\frac{2}{3} \alpha_{3}+\frac{4}{3} \alpha_{1} .
$$

Using Relations (22) and (25) in Relation (26) yields $4 A \geq N(N-1)+\frac{2}{3} N+\frac{2}{3} \alpha_{3}+\frac{4}{3} \alpha_{1}$, or $A \geq \frac{N(N-1)}{4}+\frac{N}{6}$, 
405 (ii) the number of edges is a multiple of 3.

\subsection{Constructions}

(i) the degree of each vertex is even; and

\section{Theorem 6.1 ([15])} following lemma.

as claimed. Note that to reach the equality, we need to have $\alpha_{1}=\alpha_{3}=0, \alpha_{2}=2 \alpha_{6}+\alpha_{5}$ by Relation (22), and $2 \alpha_{6}+2 \alpha_{2}+\alpha_{5}=2 N$ by Relation (25), so $\alpha_{2}=\frac{2 N}{3}$, hence an optimal decomposition should use $\frac{N}{3}$ digraphs like the digraph $G_{7}$ depicted in Figure 4, having 1 vertex of degree 6 and 2 vertices of degree 2 .

Our constructions rely on the existence of 3-GDD's, that is, decompositions of complete multipartite graphs into $K_{3}$ 's. We recall the definition and some basic results below.

Decompositions of complete multipartite graphs into $K_{3}$ 's. Let $v_{1}, v_{2}, \ldots, v_{q}$ be non-negative integers; the complete multipartite graph with group sizes $v_{1}, v_{2}, \ldots, v_{q}$ is defined to be the graph with vertex set $V_{1} \cup V_{2} \cup \cdots \cup V_{q}$ where $\left|V_{i}\right|=v_{i}$, and two vertices $u \in V_{i}$ and $v \in V_{j}$ are adjacent if $i \neq j$. Using terminology of design theory, the graph of type $p_{1}^{\alpha_{1}} p_{2}^{\alpha_{2}} \ldots p_{h}^{\alpha_{h}}$ is the complete multipartite graph with $\alpha_{i}$ groups of size $p_{i}$. The existence of a partition of this multipartite graph into $K_{k}$ 's is equivalent to the existence of a $k-G D D$ (Group Divisible Design) of type $p_{1}^{\alpha_{1}} p_{2}^{\alpha_{2}} \ldots p_{h}^{\alpha_{h}}$ (see [15]). Here we are interested in the existence of 3-GDD's, that is, partitions into $K_{3}$ 's. When $\left|V_{i}\right|=p$ for all $i$, we denote by $K_{p \times q}$ the multipartite graph of type $p^{q}$. Trivial necessary conditions for the existence of a 3-GDD are

These conditions are in general sufficient. In particular, the following results will be used later.

A 3-GDD of type $2^{q}$ with $q \geq 3$ exists if and only if $q \equiv 0$ or $1(\bmod 3)$.

A 3-GDD of type $2^{q-1} 4$ with $q \geq 4$ exists if and only if $q \equiv 1(\bmod 3)$.

A 3-GDD of type $3^{q}$ with $q \geq 3$ exists if and only if $q$ is odd.

A 3-GDD of type $3^{q-1} 1$ with $q \geq 3$ exists if and only if $q$ is odd.

A 3-GDD of type $3^{q-1} 5$ with $q \geq 5$ exists if and only if $q$ is odd.

A 3-GDD of type $3^{q-1} 11$ with $q \geq 7$ exists if and only if $q$ is odd.

The basic partition. In what follows $\vec{K}_{2,2,2}$ will denote the digraph on 6 vertices and 12 arcs depicted in Figure 5. This digraph can be viewed as being obtained from $K_{3}(i, j, k)$ with $i<j<k$ by replacing each vertex $i$ with two vertices $i_{A}$ and $i_{B}$ forming an independent set.

Note that $\vec{K}_{2,2,2}$ is an optimal digraph for $C=3$, since it attains the ratio $\rho(3)=2$ (see Table 1). The idea of the constructions consists of starting from some graph $G$ (mainly a multipartite graph) which can be decomposed into $K_{3}$ 's, replacing each vertex with two non-adjacent vertices, and then using the 


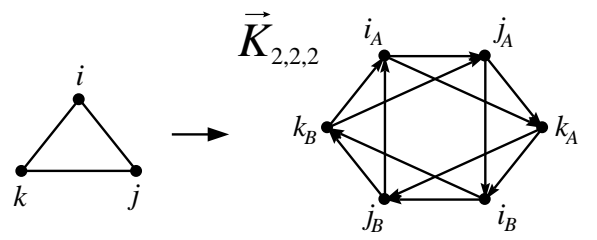

(a)

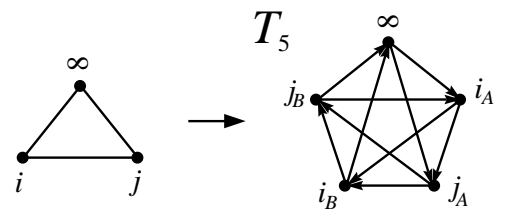

(b)

Figure 5: (a) Digraph $\vec{K}_{2,2,2}$ obtained from $K_{3}(i, j, k)$, with $i<j<k$; (b) digraph $T_{5}$ obtained from a $K_{3}$ of the form $(\infty, i, j)$.

Lemma 6.1 If a graph $G=(V, E)$ with vertex set $\{1,2, \ldots,|V|\}$ can be decomposed into $h K_{3}$ 's, then the digraph $H$ obtained from $G$ by replacing each vertex $i$ with two non-adjacent vertices $i_{A}$ and $i_{B}$, and where the vertices are ordered $1_{A}, 2_{A}, \ldots,|V|_{A}, 1_{B}, 2_{B}, \ldots,|V|_{B}$, has a valid decomposition into $\vec{K}_{2,2,2}$ 's with a total of $6 h$ vertices.

Proof: To each triangle $(i, j, k)$ with $1 \leq i<j<k \leq|V|$ is associated the $\vec{K}_{2,2,2}$ with vertices $1 \leq i_{A}<$ $j_{A}<k_{A} \leq|V|<i_{B}<j_{B}<k_{B} \leq 2|V|$. To show that the decomposition is valid for $C=3$, it suffices to show that the distance between the end-vertices of any arc of any $\vec{K}_{2,2,2}$ is at most $|V|$. That is true for the $\operatorname{arcs}\left(x_{A}, y_{A}\right)$ or $\left(x_{B}, y_{B}\right)$ as they satisfy $x<y$, and also for the arcs $\left(x_{A}, y_{B}\right)$ or $\left(x_{B}, y_{A}\right)$ as they satisfy $x>y($ see Figure 5(a)).

Some small cases. We provide here decompositions of some particular small digraphs that will be used in the constructions of Propositions 6.4 and 6.5.

Lemma 6.2 $A(3,5)=5, A(3,6) \leq 10, A(3,7) \leq 12, A(3,8) \leq 18, A(3,9) \leq 21, A(3,10) \leq 28$, $A(3,11) \leq 31$, and $A(3,23) \leq 132$.

Proof: Case $N=5$. The decomposition is given in Figure 5(b), and can be viewed as obtained from the $K_{3}(\infty, i, j)$ by replacing each of $i, j$ with two vertices.

Case $N=6,7$. The complete graph $K_{4}$ can be decomposed into one $K_{1,3}(0 ; \infty, 1,2)$ and one $K_{3}(\infty, 1,2)$. Replace each of the vertices $i, j, k$ with two vertices. The $T_{7}$ on the ordered vertices $\infty, 0_{A}, 1_{A}, 2_{A}, 0_{B}, 1_{B}, 2_{B}$ can be partitioned into a $T_{5}$ on $\infty, 1_{A}, 2_{A}, 1_{B}, 2_{B}(($ see Figure 5 (b) with $i=1, j=$ 2)) and the admissible digraph on 7 vertices and 11 arcs depicted in Figure 6(b) with $i=0, j=1, k=2$. So we obtain a valid decomposition using 12 vertices. Deleting vertex $\infty$ yields a decomposition of $T_{5}$ with 10 vertices.

Case $N=8,9 . K_{5}$ is the union of two $K_{3}$ 's $(\infty, 1,3),(0,2,3)$ and a $C_{4}(\infty, 0,1,2)$. Replacing each vertex with two vertices we get a partition of the $T_{9}$ on the ordered vertices $\infty, 0_{A}, 1_{A}, 2_{A}, 3_{A}, 0_{B}, 1_{B}, 2_{B}, 3_{B}$. Namely, to the $K_{3}(\infty, 1,3)$ we associate a $T_{5}$ on $\infty, 1_{A}, 3_{A}, 1_{B}, 3_{B}$ (see Figure 5 (b) with $i=1, j=3$ ). To the $K_{3}(0,2,3)$ we associate a $\vec{K}_{2,2,2}$ on $0_{A}, 2_{A}, 3_{A}, 0_{B}, 2_{B}, 3_{B}$. To the $C_{4}(\infty, 0,1,2)$ we associate the digraph on 7 vertices of Figure 6 (a) with $i=0, j=1, k=2$ and the triangle $\left(1_{A}, 2_{A}, 2_{B}\right)$. Therefore, $A(3,9) \leq 21$. Vertex $1_{A}$ appears in 3 digraphs, so $A(3,8) \leq 21-3=18$. 


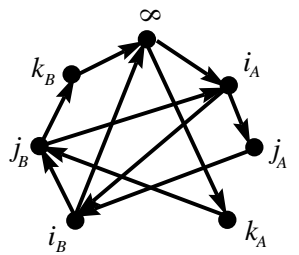

(a)

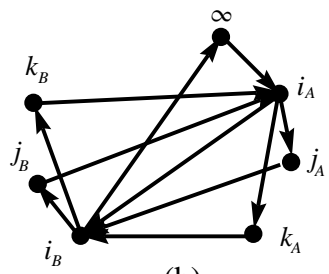

(b)

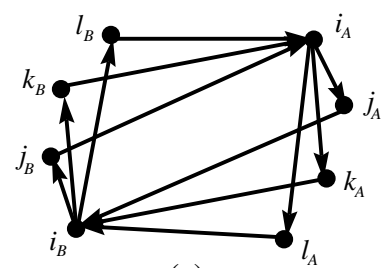

(c)

Figure 6: (a) Digraph associated to a $C_{4}(\infty, i, j, k)$. Digraphs associated to stars $\left(K_{1,3}\right.$ 's), with $\infty<i<$ $j<k<\ell$ : (b) star of the form $(i ; \infty, j, k)$; (c) star of the form $(i ; j, k, \ell)$.

Case $N=10,11 . K_{6}$ can be partitioned into $3 K_{3}$ 's $(\infty, 1,3),(\infty, 2,4),(0,1,4)$, a star $K_{1,3}(0 ; \infty, 2,3)$, and a $P_{4}[1,2,3,4]$. Replacing each vertex with two vertices we get a partition of the $T_{11}$ on the ordered vertices $\infty, 0_{A}, 1_{A}, 2_{A}, 3_{A}, 4_{A}, 0_{B}, 1_{B}, 2_{B}, 3_{B}, 4_{B}$ into $2 T_{5}$ 's on $\infty, 1_{A}, 3_{A}, 1_{B}, 3_{B}$ and $\infty, 2_{A}, 4_{A}, 2_{B}, 4_{B}$, a $\vec{K}_{2,2,2}$ on $0_{A}, 1_{A}, 4_{A}, 0_{B}, 1_{B}, 4_{B}$, a digraph on 7 vertices and 11 arcs depicted in Figure 6(b) with $i=0, j=$ $2, k=3$, and an admissible digraph on 8 vertices with $\operatorname{arcs}\left(1_{A}, 2_{A}\right),\left(2_{A}, 3_{A}\right),\left(3_{A}, 4_{A}\right),\left(1_{B}, 2_{B}\right),\left(2_{B}, 3_{B}\right),\left(3_{B}, 4_{B}\right)$, $\left(2_{A}, 1_{B}\right),\left(2_{B}, 1_{A}\right),\left(3_{A}, 2_{B}\right),\left(3_{B}, 2_{A}\right),\left(4_{A}, 3_{B}\right),\left(4_{B}, 3_{A}\right)$. Therefore, $A(3,11) \leq 31$, and as vertex $\infty$ appears in 3 subgraphs, we get $A(3,10) \leq 28$.

Case $N=23$. We decompose $K_{12}$ into $19 K_{3}$ 's and $3 K_{1,3}$ 's, where vertex $\infty$ appears in $5 K_{3}$ 's and in a $\operatorname{star}(i ; \infty, j, k)$, the two other stars being of the form $\left(i^{\prime} ; j^{\prime} k^{\prime}, \ell^{\prime}\right)$ with $i^{\prime}<j^{\prime}<k^{\prime}<\ell^{\prime}$. We obtain a decomposition of $T_{23}$ into $5 T_{5}$ 's, $14 \vec{K}_{2,2,2}$ 's, 1 digraph of Figure 6(a), and 2 digraphs of Figure 6(c). Thus, $A(3,23) \leq 5 \cdot 5+14 \cdot 6+7+8+8=132$.

Constructions. We begin with an optimal partition for $N \equiv 0,1,4$, or $5(\bmod 12)$, and then we provide near-optimal constructions for the remaining values.

\section{Proposition 6.3}

If $N \equiv 0$ or $4(\bmod 12), A(3, N)=\frac{N^{2}}{4}$.

If $N \equiv 1$ or $5(\bmod 12), A(3, N)=\frac{N(N-1)}{4}$.

Proof: The lower bound follows from Propositions 6.1 and 6.2. For the upper bound, we will apply Lemma 6.1 with $G=K_{2 \times q}$ (type $2^{q}$ ), which can be decomposed by Theorem 6.1 into $\frac{2 q(q-1)}{3} K_{3}$ 's if $q \equiv 0$ or $1(\bmod 3)$. As $G$ has $2 q$ vertices, the graph $H$ described in Lemma 6.1 has $4 q$ vertices and can be decomposed into admissible $\vec{K}_{2,2,2}$ 's. Adding an admissible $T_{4}$ on each of the $q$ independent sets of $H$ (of the form $\left\{i_{A}, j_{A}, i_{B}, j_{B}\right\}$ where $\{i, j\}$ is an independent set of $\left.G\right)$, we get a valid decomposition of $T_{4 q}$ into $q T_{4}$ 's and $\frac{2 q(q-1)}{3}$ admissible $\vec{K}_{2,2,2}$ 's. So using $A(3,4)=4$, we get $A(3,4 q) \leq q A(3,4)+4 q(q-1)=4 q^{2}$ for $q \equiv 0$ or $1(\bmod 3)$. So $A(3, N) \leq \frac{N^{2}}{4}$ for $N \equiv 0$ or $4(\bmod 12)$.

For $N=4 q+1$, we add to the vertex set of $H$ an extra vertex $\infty$. Adding to the arcs of $H$ the $q$ tournaments $T_{5}$ built on $\infty, i_{A}, j_{A}, i_{B}, j_{B}$, where vertices $i, j$ are not adjacent in $G$, we get a decomposition 
of $T_{4 q+1}$ into $q$ admissible $T_{5}$ 's plus $\frac{2 q(q-1)}{3}$ admissible $\vec{K}_{2,2,2}$ 's (the distance being at most $2 q-1$ in $H$ and so $2 q$ in $\left.T_{4 q+1}\right)$. Using $A(3,5)=5$ (see Lemma 6.2), we get $A(3,4 q+1) \leq q A(3,5)+4 q(q-1)=$ $4 q^{2}+q=\frac{(4 q+1) 4 q}{4}$ for $q \equiv 0$ or $1(\bmod 3)$. So $A(3, N) \leq \frac{N(N-1)}{4}$ for $N \equiv 1$ or $5(\bmod 12)$.

We group the non-optimal constructions in Proposition 6.4 and Proposition 6.5 according to whether they differ from the lower bound by either a constant or a linear additive term, respectively.

\section{Proposition 6.4}

If $N \equiv 8(\bmod 12), \quad A(3, N) \leq \frac{N^{2}}{4}+2$.

If $N \equiv 9(\bmod 12), \quad A(3, N)=\frac{N(N-1)}{4}+3$.

Proof: We start from $G$ of type $2^{q-1} 4$ with $q \equiv 1(\bmod 3)$, which can be decomposed by Lemma 6.1 into $\frac{2(q-1)(q+2)}{3} K_{3}$ 's. As in the proof of Proposition 6.3, we get a decomposition of $T_{4 q+4}$ into $q-1 T_{4}$ 's, one $T_{8}$ and $\frac{2(q-1)(q+2)}{3} \vec{K}_{2,2,2}$ 's (indeed, the independent set $V_{q}$ of $G$ has 4 vertices, so in $H$ it induces an independent set of 8 vertices). So using $A(3,4)=4$ and $A(3,8) \leq 18$ (see Lemma 6.2), we get $A(3,4 q+4) \leq(q-1) A(3,4)+A(3,8)+4(q-1)(q+2) \leq 4 q^{2}+8 q+6=\frac{(4 q+4)^{2}}{4}+2$ for $q \equiv 1(\bmod 3)$, so $A(3, N) \leq \frac{N^{2}}{4}+2$ for $N \equiv 8(\bmod 12)$.

Similarly, adding a vertex $\infty$ to $H$ we get a decomposition of $T_{4 q+1}$ into $q-1 T_{5}$ 's, one $T_{9}$ and $h=\frac{2(q-1)(q+2)}{3} K_{3}$ 's. So using $A(3,5)=5$ and $A(3,9) \leq 21$ we get $A(3,4 q+5) \leq(q-1) A(3,5)+A(3,9)+$ $4(q-1)(q+2) \leq 4 q^{2}+9 q+8=\frac{(4 q+5)(4 q+4)}{4}+3$ for $q \equiv 1(\bmod 3)$, so $A(3, N) \leq \frac{N(N-1)}{4}+3$ for $N \equiv 9$ $(\bmod 12)$.

\section{Proposition 6.5}

If $N \equiv 2(\bmod 12), \quad A(3, N) \leq \frac{N^{2}}{4}+\frac{N+4}{6}$.

If $N \equiv 3(\bmod 12), \quad A(3, N) \leq \frac{N^{2}+3}{4}$.

If $N \equiv 6(\bmod 12), \quad A(3, N) \leq \frac{N^{2}}{4}+\frac{N}{6}$.

If $N \equiv 7(\bmod 12), \quad A(3, N) \leq \frac{N^{2}-1}{4}$.

If $N \equiv 10(\bmod 12), \quad A(3, N) \leq \frac{N^{2}}{4}+\frac{N+8}{6}$.

If $N \equiv 11(\bmod 12), A(3, N) \leq \frac{N^{2}+3}{4}+\varepsilon$, with $\varepsilon=1$ for $N=11,35$.

Proof: We use as graph $G$ of Lemma 6.1 a multipartite graph of type $3^{q-1} u$ with $3(q-1)+u$ vertices, in order to get a decomposition of $T_{6(q-1)+2 u}$ (resp. $\left.T_{6(q-1)+2 u+1}\right)$ into $q-1 T_{6}$ 's (resp. $T_{7}$ 's), one $T_{2 u}$ (resp. $\left.T_{2 u+1}\right)$ and the digraph $H$ itself decomposed by Lemma 6.1 into $h=\frac{9(q-1)(q-2)}{6}+u(q-1) \vec{K}_{2,2,2}$ 's. We distinguish several cases according to the value of $u$.

Case 1: $u=1, q \geq 3$ odd.

Let $N \equiv 2(\bmod 12), N=6 q-4$. Using $A(3,2)=2$ and $A(3,6) \leq 10$ we get $A(3,6 q-4) \leq$ $(q-1) A(3,6)+A(3,2)+(q-1)(9 q-12) \leq 9 q^{2}-11 q+4=\frac{(6 q-4)^{2}}{4}+q=\frac{N^{2}}{4}+\frac{N+4}{6}$.

Let $N \equiv 3(\bmod 12), N=6 q-3$. Using $A(3,3)=3$ and $A(3,7) \leq 12$ we get $A(3,6 q-3) \leq$ $(q-1) A(3,7)+A(3,3)+(q-1)(9 q-12) \leq 9 q^{2}-9 q+3=\frac{(6 q-3)^{2}}{4}+\frac{3}{4}=\frac{N^{2}+3}{4}$.

Case 2: $u=3, q \geq 3$ odd. 
Let $N \equiv 6(\bmod 12), N=6 q$. Using $A(3,6) \leq 10$ we get $A(3,6 q) \leq q A(3,6)+9 q(q-1) \leq 9 q^{2}+q=$ $\frac{N^{2}}{4}+\frac{N}{6}$.

Let $N \equiv 7(\bmod 12), N=6 q+1$. Using $A(3,7) \leq 12$ we get $A(3,6 q+1) \leq q A(3,7)+9 q(q-1) \leq$ $9 q^{2}+3 q=\frac{N^{2}-1}{4}$.

Case 3: $u=5, q \geq 5$ odd.

Let $N \equiv 10(\bmod 12), N=6 q+4$. Using $A(3,6) \leq 10$ and $A(3,10) \leq 28$ we get $A(3,6 q+4) \leq$ $(q-1) A(3,6)+A(3,10)+(q-1)(9 q+12) \leq 9 q^{2}+13 q+6=\frac{(6 q+4)^{2}}{4}+\frac{6 q+12}{6}=\frac{N^{2}}{4}+\frac{N+8}{6}$.

Let $N \equiv 11(\bmod 12), N=6 q+5$. Using $A(3,7) \leq 12$ and $A(3,11) \leq 31$ we get $A(3,6 q+5) \leq$ $(q-1) A(3,7)+A(3,11)+(q-1)(9 q+12) \leq 9 q^{2}+15 q+7=\frac{N^{2}+3}{4}$.

For $q=23$ we have $A(3,23) \leq 132=\frac{23^{2}-1}{4}$, one less than the value given by the preceding construction. Using $u=11, q \geq 7$ odd, $N=6 q+17, A(3,7) \leq 12$, and $A(3,23) \leq 132$ we get $A(3,6 q+17) \leq(q-1) A(3,7)+A(3,23)+(q-1)(9 q+48) \leq 9 q^{2}+51 q+72=\frac{(6 q+17)^{2}-1}{4}=\frac{N^{2}-1}{4}$. It might be that $A(3,11) \leq 30$, and then the bound $\frac{N^{2}-1}{4}$ would be also attained for $N=11$ and 35 .

\section{Case $C>3$}

For $C>3$, we distinguish two cases according to whether $C$ is of the form $\frac{k(k+1)}{2}$ or not. We focus on those cases in Sections 7.1 and 7.2.

\section{1 $C$ not of the form $k(k+1) / 2$}

If $C$ is not of the form $\frac{k(k+1)}{2}$, we can improve the lower bound of Theorem 3.1, as we did for $C=2$ in Proposition 5.1. We provide the details for $C=4$ and sketch the ideas for $C=5$, that show how to improve the lower bound for any value of $C$ not of the form $k(k+1) / 2$.

\section{Proposition 7.1}

$$
A(4, N) \geq \frac{7}{32} N(N-1)=\left(\frac{3}{14}+\frac{1}{224}\right) N(N-1) .
$$

Proof: The values of $\gamma(4, p)$ are given in Table 1, so Relation (13) becomes in the case $C=4$ $A=\sum_{p=2}^{N} p a_{p} \geq \frac{3}{7} \sum_{p=2}^{N} a_{p} \gamma(4, p)+\frac{11}{7} a_{2}+\frac{12}{7} a_{3}+\frac{10}{7} a_{4}+\frac{5}{7} a_{5}+\frac{3}{7} a_{6}+\frac{1}{7}\left(a_{7}+2 a_{8}+a_{10}+2 a_{11}+a_{13}+2 a_{14}+\cdots\right)$. Using $\sum_{p=2}^{N} a_{p} \gamma(4, p) \geq \frac{N(N-1)}{2}$, Relation (27) becomes

$$
14 A \geq 3 N(N-1)+22 a_{2}+24 a_{3}+20 a_{4}+10 a_{5}+6 a_{6}+2 a_{7}+4 a_{8}+\cdots
$$

On the other hand,

$$
A \geq 9\left(W-\sum_{i=2}^{8} a_{i}\right)+\sum_{i=2}^{8} i \cdot a_{i}=9 W-7 a_{2}-6 a_{3}-5 a_{4}-4 a_{5}-3 a_{6}-2 a_{7}-a_{8} .
$$


Summing Relations (28) and (29) and using $W \geq \frac{N(N-1)}{32}+\frac{N-1}{32}$ by Proposition 3.1 yields

$$
15 A \geq \frac{105}{32} N(N-1)+\frac{9}{32}(N-1), \text { and therefore } A \geq \frac{7}{32} N(N-1)+\frac{3}{160}(N-1) .
$$

For $C=5$, a similar computation with $\rho(5)=8 / 3$ gives

$$
\begin{array}{r}
8 A \geq \frac{3}{2} N(N-1)+13 a_{2}+15 a_{3}+14 a_{4}+10 a_{5}+3 a_{6}+2 a_{7}+a_{8} . \\
A \geq 9 W-7 a_{2}-6 a_{3}-5 a_{4}-4 a_{5}-3 a_{6}-2 a_{7}-a_{8} .
\end{array}
$$

So again, summing Relations (30) and (31) and using $W \geq \frac{N(N-1)}{40}+\frac{N-1}{40}$ by Proposition 3.1 yields

$$
A \geq \frac{N(N-1)}{6}+\frac{N(N-1)}{40}+\frac{N-1}{40}=\frac{23}{120} N(N-1)+\frac{N-1}{40}=\left(\frac{3}{16}+\frac{1}{240}\right) N(N-1)+\frac{N-1}{40} .
$$

\section{2 $C$ of the form $k(k+1) / 2$}

For $C=\frac{k(k+1)}{2}$ the lower bound of Theorem 3.1 can be attained, according to the existence of a type of $k-G D D$, called a Balanced Incomplete Block Design $(B I B D)$. A $(v, k, 1)-B I B D$ consists simply of a partition of $K_{v}$ into $K_{k}$ 's.

Theorem 7.1 If there exists a $(k+1)-G D D$ of type $k^{q}$ (that is, a decomposition of $K_{k \times q}$ into $K_{k+1}$ 's), then there exists an optimal admissible partition of $T_{2 k q+1}$ for $C=\frac{k(k+1)}{2}$ with $\frac{N(N-1)}{2 k} A D M s$.

Proof: The lower bound follows from Theorem 3.1. For the upper bound, as we did in Proposition 6.3 (case $k=2, C=2$ ), we replace each vertex $i$ of $K_{k \times q}$ with two vertices $i_{A}$ and $i_{B}$, and add a new vertex $\infty$. We label the vertices of the obtained $T_{2 k q+1}$ with $\infty, 1_{A}, \ldots,(k q)_{A}, 1_{B}, \ldots,(k q)_{B}$. To each $K_{k+1}$ of the decomposition of $K_{k \times q}$ we associate a $T_{2 \times(k+1)}$, which is an optimal digraph for $C=\frac{k(k+1)}{2}$ with $2(k+1)$ vertices and $2 k(k+1)$ edges, hence attaining $\rho(C)=k$. So adding vertex $\infty$ to the stable sets of size $2 k$ we obtain a decomposition of $T_{2 k q+1}$ into $q T_{2 k+1}$ 's (which are also optimal) and $T_{2 \times(k+1)}$ 's.

If $K_{k \times q}$ is decomposable into $K_{k+1}$ 's, the number of $K_{k+1}$ 's (and so the number of $T_{2 \times(k+1)}$ 's) is $\frac{k q(q-1)}{k+1}$. Therefore the total number of ADMs is $q(2 k+1)+2 k q(q-1)=\frac{(2 k q+1) 2 k q}{2 k}=\frac{N(N-1)}{2 k}$.

Note that a decomposition of $K_{k \times q}$ into $K_{k+1}$ 's is equivalent to a decomposition of $K_{k q+1}$ into $K_{k+1}$ 's by adding a new vertex $\infty$, that is, a $(k q+1, k+1,1)$-BIBD. In particular, such designs are known to exist if $N$ is large enough and $(k q+1) k q \equiv 0(\bmod k(k+1))$ [15]. For example, for $k=3$ and $q \equiv 0$ or 1 $(\bmod 4)$, or $k=4$ and $q \equiv 0$ or $1(\bmod 5)$.

\section{Corollary 7.1}

If $C=6$ and $N \equiv 1$ or $7(\bmod 24), A(6, N)=\frac{N(N-1)}{6}$.

If $C=10$ and $N \equiv 1$ or $9(\bmod 40), A(10, N)=\frac{N(N-1)}{8}$. 
Corollary 7.2 For $C \in\{15,21,28,36\}$, there exists a small set of values of $N$ for which the existence of a $B I B D$ remains undecided (179 values overall, see [15, pages 73-74]). For the values of $N$ different from these undecided BIBDs, the following results apply.

If $C=15$ and $N \equiv 1$ or $11(\bmod 30), A(15, N)=\frac{N(N-1)}{10}$.

If $C=21$ and $N \equiv 1$ or $13(\bmod 84), A(21, N)=\frac{N(N-1)}{12}$.

If $C=28$ and $N \equiv 1$ or $15(\bmod 112), A(28, N)=\frac{N(N-1)}{14}$.

If $C=36$ and $N \equiv 1$ or $17(\bmod 144), A(36, N)=\frac{N(N-1)}{16}$.

Wilson proved [34] that for $v$ large enough, $K_{v}$ can be decomposed into subgraphs isomorphic to any given graph $G$, if the trivial necessary conditions about the degree and the number of edges are satisfied. Thus, we can assure that optimal constructions exist when $C=\frac{k(k+1)}{2}$ for all $k>0$.

Corollary 7.3 If $C=\frac{k(k+1)}{2}$, then $A(C, N)=\frac{N(N-1)}{2 k}$ for $N \equiv 1$ or $2 k+1(\bmod 4 C)$ large enough.

We can also use decompositions of $K_{p \times q}$ into $K_{k+1}$ 's to get constructions asymptotically optimal, but not attaining the lower bound like for $C=3$. For instance, for $C=6$ the proof of Theorem 7.1 gives (without adding the vertex $\infty)$ that for $q \equiv 0$ or $1(\bmod 4)$ and $N \equiv 0$ or $6(\bmod 24)$,

$$
A(6,6 q) \leq q A(6,6)+6 q(q-1)=6 q^{2}=\frac{N^{2}}{6} .
$$

That might be an optimal value if we could improve the lower bound for $C=6$ as we $\operatorname{did}$ for $C=3$ in Proposition 6.2, but the calculations become considerably more complicated.

\section{Corollary 7.4}

For $N \equiv 0$ or $6(\bmod 24), \frac{N(N-1)}{6} \leq A(6, N) \leq \frac{N^{2}}{6}$.

For $N \equiv 0$ or $8(\bmod 40), \frac{N(N-1)}{8} \leq A(10, N) \leq \frac{N^{2}}{8}$.

For a general $C$ of the form $C=\frac{k(k+1)}{2}$, the improved lower bound one could expect is $\frac{N^{2}}{2 k}$.

Finally, it is worth mentioning here the constructions given in [19] for $C=8$. Namely, in [19, Corollary 5] the authors provide a construction that uses asymptotically $\frac{N^{2}}{2} \frac{5}{16} \mathrm{ADMs}$, using the socalled primitive rings. This construction, according to the lower bound of Theorem 3.1, constitutes a $\frac{35}{32}$ approximation for $C=8$. Note that the construction for $C=6$ given in Corollary 7.1 uses asymptotically $\frac{N^{2}}{2} \frac{1}{3}=\frac{N^{2}}{2} \frac{5}{15}$ ADMs, which is already very close to the value obtained in [19] for $C=8$, so it seems natural to suspect that there is enough room for improvement over the constructions of [19].

\section{Unidirectional or Bidirectional Rings?}

This section is devoted to comparing unidirectional and bidirectional rings in terms of minimizing electronics cost, when these rings are used in a WDM network with traffic grooming and all-to-all requests. 
For bidirectional rings, Theorem 3.1 gives the following lower bound by multiplying by 2 the value, in order to take into account requests both clockwise and counterclockwise.

$$
\mathrm{LB}_{\mathrm{bi}}(C, N)=\frac{N(N-1)}{2} \cdot \frac{2}{\rho(C)},
$$

where $\rho(C)=k+\frac{r}{k+1}$ for $C=\frac{k(k+1)}{2}+r$ with $0 \leq r \leq k$.

In [7] the following general lower bound was given for unidirectional rings.

$$
\begin{gathered}
\mathrm{LB}_{\text {uni }}(C, N)=\frac{N(N-1)}{2} \cdot \frac{1}{\eta(C)}, \\
\text { where } \eta(C)=\left\{\begin{array}{cl}
\frac{k}{2}, & \text { if } C=\frac{k(k+1)}{2}+r \text { and } 0 \leq r \leq \frac{k}{2} \\
\frac{C}{k+2}, & \text { if } C=\frac{k(k+1)}{2}+r \text { and } \frac{k}{2} \leq r \leq k
\end{array}\right.
\end{gathered}
$$

Note that for $C=\frac{k(k+1)}{2}$ (that is, for $r=0$ ) the bounds are equal. In general, we have

$$
1 \leq \frac{\mathrm{LB}_{\mathrm{uni}}(C, N)}{\mathrm{LB}_{\mathrm{bi}}(C, N)} \leq 1+\frac{1}{2(k+1)}
$$

Indeed, either $0 \leq r \leq \frac{k}{2}$ and then

$$
\frac{\rho(C)}{2 \eta(C)}=1+\frac{r}{k(k+1)} \leq 1+\frac{1}{2(k+1)},
$$

or $\frac{k}{2} \leq r \leq k$, and then

$$
\frac{\rho(C)}{2 \eta(C)}=\frac{(k+2)(k(k+1)+r)}{(k+1)(k(k+1)+2 r)}=1+\frac{k(k+1)-r k}{(k+1)(k(k+1)+2 r)} .
$$

Let $r=\frac{k}{2}+r^{\prime}$, and so $0 \leq r^{\prime} \leq \frac{k}{2}$. Then

$$
\frac{\rho(C)}{2 \eta(C)}=1+\frac{1}{2(k+1)} \frac{k(k+2)-2 r^{\prime}}{k(k+2)+2 r^{\prime}} \leq 1=\frac{1}{2(k+1)} .
$$

Note that there exist constructions for bidirectional rings with cost strictly smaller than $\operatorname{LB}_{\text {uni }}(C, N)$. Indeed, for $C=2$ we presented in Section 5.2.2 a construction using at most $\frac{17}{48} N(N-1)$ ADMs. Taking into account requests in both directions this construction uses at most $\frac{17}{24} N(N-1)$ ADMs, to be compared with $\mathrm{LB}_{\text {uni }}(2, N)=\frac{3}{4} N(N-1)>\frac{17}{24} N(N-1)$.

However, for large $C$ the lower bounds tend to be equal; hence in terms of the number of ADMs there is no real improvement in using bidirectional rings. The real improvement is more in terms of the number of used wavelengths (or, equivalently, the load). Indeed, in unidirectional rings this number is roughly $\frac{N^{2}}{2 C}$ (see for instance [7]), which is twice the number in bidirectional rings (roughly equal to $2 \cdot \frac{N^{2}}{8 C}$ by Proposition 3.1). 
In summary, bidirectional and unidirectional rings are equivalent in terms of the number of ADMs, the trade-off being between better bandwidth utilization in bidirectional rings versus simplicity (and the use of the other ring for fault tolerance) in unidirectional rings.

\section{Conclusions and Further Research}

In this article we studied the minimization of ADMs in optical WDM bidirectional ring networks under the assumption of symmetric shortest path routing and all-to-all unitary requests. We precisely formulated the problem in terms of graph decompositions, and stated a general lower bound for all the values of $C$ and $N$. We then studied extensively the cases $C=2$ and $C=3$, providing improved lower bounds, optimal constructions for several infinite families, as well as asymptotically optimal constructions and approximations. To the best of our knowledge, these are the first optimal solutions in the literature for traffic grooming in bidirectional rings. We then study the case $C>3$, focusing specifically on the case $C=k(k+1) / 2$ for some $k \geq 1$. We gave optimal decompositions for several congruence classes of $N$, using the existence of some combinatorial designs. We concluded with a comparison of the switching cost in unidirectional and bidirectional WDM rings.

Further research is needed to find new families of optimal solutions for other values of $C$. The first step should be to improve the general lower bound for other values of $C$, namely, finding a closed formula. It would be interesting to consider other kinds of routing in bidirectional rings, not necessarily symmetric or using shortest paths. Stating which kind of routing is the best for each value of $N$ and $C$ would be a nice result. Finally, studying the traffic grooming problem using graph partitioning tools in other topologies, like trees or hypercubes, would be also interesting.

Acknowledgement. We would like to thank D. Coudert, T. Chow, and P. Lin for insightful discussions.

\section{References}

[1] O. Amini, S. Pérennes, and I. Sau, Hardness and approximation of traffic grooming, Theoret Comput Sci 410 (2009), 3751-3760.

[2] B. Beauquier, J.C. Bermond, L. Gargano, P. Hell, S. Pérennes, and U. Vaccaro, Graph problems arising from wavelength-routing in all-optical networks, IEEE Workshop Optics Comput Sci (WOCS), 1997, pp. 1-12.

[3] J.C. Bermond, L. Braud, and D. Coudert, Traffic grooming on the path, Theoret Comput Sci 384 (2007), 139-151.

[4] J.C. Bermond, C. Colbourn, D. Coudert, G. Ge, A. Ling, and X. Muñoz, Traffic grooming in unidirectional WDM rings with grooming ratio C=6, SIAM J Discr Math 19 (2005), 523-542. 
[5] J.C. Bermond, C. Colbourn, A. Ling, and M.L. Yu, Grooming in unidirectional rings: $K_{4}-e$ designs, Discr Math 284 (2004), 57-62.

[6] J.C. Bermond, M. Cosnard, D. Coudert, and S. Pérennes, Optimal solution of the maximum all request path grooming problem, Advanced Int Conference Telecommunications (AICT), IEEE 2006, pp. 1-6.

[7] J.C. Bermond and D. Coudert, Traffic grooming in unidirectional WDM ring networks using design theory, IEEE Int Conference Commun (ICC), Vol. 2, 2003, pp. 1402-1406.

[8] J.C. Bermond and D. Coudert, The CRC Handbook of Combinatorial Designs (2nd edition) Vol. 42 of Discrete Mathematics and Its Applications chapter VI.27, Grooming, CRC Press C.J. Colbourn and J.H. Dinitz edition 2006.

[9] J.C. Bermond, D. Coudert, X. Muñoz, and I. Sau, Traffic grooming in bidirectional WDM ring networks, IEEE-LEOS ICTON / COST 293 GRAAL, Vol. 3, 2006, pp. 19-22.

[10] J.C. Bermond, D. Coudert, and M.L. Yu, On DRC-covering of $K_{n}$ by cycles, J Combinatorial Designs 11 (2003), 100-112.

[11] R. Berry and E. Modiano, Reducing electronic multiplexing costs in SONET/WDM rings with dynamically changing traffic, IEEE J Selected Areas in Commun (2000), 1961-1971.

[12] A. Chiu and E. Modiano, Traffic grooming algorithms for reducing electronic multiplexing costs in WDM ring networks, IEEE/OSA J Lightwave Technology 18 (2000), 2-12.

[13] T.Y. Chow and P.J. Lin, private communication.

[14] T.Y. Chow and P.J. Lin, The ring grooming problem, Networks 44 (2004), 194-202.

[15] C.J. Colbourn and J. Dinitz (Editors), The CRC Handbook of Combinatorial Designs (2nd edition) Vol. 42, CRC Press, 2006.

[16] C.J. Colbourn, H.L. Fu, G. Ge, A.C.H. Ling, and H.C. Lu, Minimizing SONET ADMs in unidirectional WDM rings with grooming ratio seven, SIAM J Discr Math 23 (2008), 109-122.

[17] C.J. Colbourn, G. Ge, and A.C.H. Ling, Optical grooming with grooming ratio eight, Discr Appl Math 157 (2009), 2763-2772.

[18] C.J. Colbourn and A.C.H. Ling, Graph decompositions with application to wavelength add-drop multiplexing for minimizing SONET ADMs, Discr Math 261 (2003), 141-156.

[19] C.J. Colbourn and P.J. Wan, Minimizing drop cost for SONET/WDM networks with $\frac{1}{8}$ wavelength requirements, Networks 37 (2001), 107-116.

[20] R. Dutta and G. Rouskas, A survey of virtual topology design algorithms for wavelength routed optical networks, Optical Networks 1 (2000), 73-89.

[21] R. Dutta and G. Rouskas, On optimal traffic grooming in WDM rings, ACM Sigmetrics/Performance, Cambridge, 2001, pp. 164-174. 
[22] R. Dutta and G. Rouskas, Traffic grooming in WDM networks: Past and future, IEEE Network 16 (2002), 46-56.

[23] O. Gerstel, P. Lin, and G. Sasaki, Wavelength assignment in a WDM ring to minimize cost of embedded sonet rings, IEEE INFOCOM (1998), 94-101.

[24] O. Gerstel, P. Lin, and G. Sasaki, Combined WDM and SONET network design, IEEE INFOCOM, 1999, pp. 734-743.

[25] O. Gerstel, R. Ramaswami, and G. Sasaki, Cost effective traffic grooming in WDM rings, IEEE INFOCOM, 1998, pp. 69-77.

[26] J. Hu, Traffic grooming in WDM ring networks: A linear programming solution, OSA J Optical Networks 1 (2002), 397-408.

[27] X.Y. Li and P.J. Wan, Select line speeds for single-hub SONET/WDM ring networks, IEEE Int Conference Commun (ICC), 2000, pp. 495-499.

[28] Z. Li and I. Sau, Graph partitioning and traffic grooming with bounded degree request graph, 35th Int Workshop Graph-Theoretic Concepts in Comput Sci (WG), Vol. 5911 of Lecture Notes in Computer Science, 2009, pp. 250-261.

[29] E. Modiano and P. Lin, Traffic grooming in WDM networks, IEEE Commun Magazine 39 (2001), $124-129$.

[30] X. Muñoz and I. Sau, Traffic grooming in unidirectional WDM rings with bounded-degree request graph, 34th Int Workshop Graph-Theoretic Concepts in Comput Sci (WG), Vol. 5344 of Lecture Notes in Computer Science, 2008, pp. 300-311.

[31] A. Somani, Survivable traffic grooming in WDM networks, Broad Band Optical Fiber Commun Technology (BBOFCT), Jalgaon, India. Nirtali Prakashan, 2001, pp. 17-45.

[32] P.J. Wan, Multichannel optical networks: Network theory and applications, Kluwer Academic Press, 1999.

[33] P.J. Wan, G. Calinescu, L. Liu, and O. Frieder, Grooming of arbitrary traffic in SONET/WDM BLSRs, IEEE J Selected Areas in Commun 18 (2000), 1995-2003.

[34] R. Wilson, Decomposition of complete graphs into subgraphs isomorphic to a given graph, Congress numerantium 15 (1976), 647-659.

[35] K. Zhu and B. Mukherjee, A review of traffic grooming in WDM optical networks: Architectures and challenges, Optical Networks 4 (2003), 55-64. 